\title{
Cdc42 and Rab8a are critical for intestinal stem cell division, survival, and differentiation in mice
}

\author{
Ryotaro Sakamori, ${ }^{1}$ Soumyashree Das, ${ }^{1}$ Shiyan Yu, ${ }^{1}$ Shanshan Feng, ${ }^{2}$ Ewa Stypulkowski, ${ }^{1}$ \\ Yinzheng Guan, ${ }^{1}$ Veronique Douard, ${ }^{3}$ Waixing Tang, ${ }^{4}$ Ronaldo P. Ferraris, ${ }^{3}$ \\ Akihiro Harada, ${ }^{5}$ Cord Brakebusch, ${ }^{6}$ Wei Guo, ${ }^{2}$ and Nan Gao ${ }^{1,7}$
}

\begin{abstract}
1Department of Biological Sciences, Rutgers University, Newark, New Jersey, USA. 'Department of Biology, University of Pennsylvania, Philadelphia, Pennsylvania, USA. ${ }^{3}$ Department of Pharmacology and Physiology,

University of Medicine \& Dentistry of New Jersey — New Jersey Medical School, Newark, New Jersey, USA. ${ }^{4}$ Department of Pathology, Temple University School of Medicine, Philadelphia, Pennsylvania, USA. ${ }^{5}$ Department of Cell Biology, Graduate School of Medicine, Osaka University, Osaka, Japan. ${ }^{6}$ Institute of Molecular Pathology, University of Copenhagen, Copenhagen, Denmark. ${ }^{7}$ Cancer Institute of New Jersey, New Jersey, USA.
\end{abstract}

\begin{abstract}
The constant self renewal and differentiation of adult intestinal stem cells maintains a functional intestinal mucosa for a lifetime. However, the molecular mechanisms that regulate intestinal stem cell division and epithelial homeostasis are largely undefined. We report here that the small GTPases Cdc42 and Rab8a are critical regulators of these processes in mice. Conditional ablation of $C d c 42$ in the mouse intestinal epithelium resulted in the formation of large intracellular vacuolar structures containing microvilli (microvillus inclusion bodies) in epithelial enterocytes, a phenotype reminiscent of human microvillus inclusion disease (MVID), a devastating congenital intestinal disorder that results in severe nutrient deprivation. Further analysis revealed that $C d c 42$-deficient stem cells had cell division defects, reduced capacity for clonal expansion and differentiation into Paneth cells, and increased apoptosis. $C d c 42$ deficiency impaired Rab8a activation and its association with multiple effectors, and prevented trafficking of Rab8a vesicles to the midbody. This impeded cytokinesis, triggering crypt apoptosis and disrupting epithelial morphogenesis. Rab8a was also required for Cdc42-GTP activity in the intestinal epithelium, where continued cell division takes place. Furthermore, mice haploinsufficient for both $C d c 42$ and Rab8 $a$ in the intestine demonstrated abnormal crypt morphogenesis and epithelial transporter physiology, further supporting their functional interaction. These data suggest that defects of the stem cell niche can cause MVID. This hypothesis represents a conceptual departure from the conventional view of this disease, which has focused on the affected enterocytes, and suggests stem cell-based approaches could be beneficial to infants with this often lethal condition.
\end{abstract}

\section{Introduction}

Malabsorptive and maldigestive diseases are the major causes of morbidity and mortality on a global scale, causing millions of deaths per year, particularly in infants in the first year of life. The cause of nearly half of these diseases is unknown. Microvillus inclusion disease (MVID) is one of the most devastating congenital intestinal disorders and the leading cause of secretory diarrhea in newborns. The early onset of severe diarrhea triggers immediate nutrient deprivation, and most infants die within 3 to 9 months. The definitive diagnosis of MVID relies on small intestinal biopsy and EM detection of characteristic inclusion bodies, the inner surfaces of which are lined by typical microvilli (1). No medication is available so far to prevent or treat this disease, thus the current therapeutic intervention for MVID is solely dependent on intravenous nutrient administration. Recent human and mouse genetic studies have revealed two genes associated with this disease. Nonsense or missense mutations of $M Y O 5 B$, a gene encoding for a myosin motor protein, are found in a group of MVID patients (2). As Myo5B is a downstream effector of Rab8a (3), the identification of a MVID-like phenotype in Rab8a-knockout mouse intestine (4) indicates that a disease-related molecular pathway is emerging

Conflict of interest: The authors have declared that no conflict of interest exists. Citation for this article: J Clin Invest. 2012;122(3):1052-1065. doi:10.1172/JCI60282. involving intracellular protein traffic. However, the underlying disease mechanism is yet to be identified.

Rab8a is a small GTPase of the Rab subfamily and has been well conserved from yeast to humans according to protein sequence homology (5). During intestinal epithelial differentiation, Rab8a has been identified as one of the key protein trafficking mediators regulated by $\mathrm{Cdx} 2$ (6), the intestinal master regulator (7). Surprisingly, although in vitro cell culture studies demonstrate that Rab8a regulates basolateral trafficking (8-10) and de novo lumen formation (11) in the MDCK kidney cell line, none of these defects is manifested in Rab8a homozygous knockout mouse kidney, which demonstrates normal renal epithelial polarity and structure (4). Instead, the intestinal epithelium of Rab8a-knockout mice shows a microvillus inclusion phenotype, leading to the death of all homozygous knockout animals within 3 to 4 weeks of age (4). These observations advocate for a careful search of tissue-specific physiological characteristics that make the intestinal epithelium more vulnerable or sensitive to trafficking disturbance.

The entire mammalian intestinal mucosa is continuously replenished by self-renewing stem cells in the crypts every day (12). Genetic tracing of $\mathrm{Lgr}^{+}$intestinal stem cells illustrates a neutral competition mechanism by which clonal expansion of a single stem cell leads to crypt homeostasis in adult intestines (13). After stochastically adopting a transit-amplifying fate, some stem cell 
descendants give rise to the polarized enterocytes, goblet cells, and enteroendocrine cells that migrate up to the villi. Other cells terminally differentiate into Paneth cells, which intersperse with stem cells at the crypt bottom (12). Recent coculture and cell ablation studies revealed that Paneth cells provide essential niche signals to support stem cell survival and renewal (12), suggesting that individual stem cells compete for niche signals via certain mechanisms yet to be discovered. Thus, the intrinsic cellular machinery that coordinates stem cell division, expansion, and terminal differentiation is largely undefined.

In this study, we report that the small GTPase Cdc42 regulates intestinal stem cell homeostasis. Using conditional gene ablation and genetic tracing, we demonstrate that intestine-specific $C d c 42-$ deficient mice develop MVID, which recapitulates the human disease counterpart. In addition to the Rab8a-knockout mice, these Cdc42-deficient mice represent the second MVID animal model confirmed at both EM and physiological levels. We find that Cdc42 is essential for midbody trafficking of Rab8a vesicle during cytokinesis. Rab8a GTP levels are significantly abrogated in Cdc42-deficient intestines in vivo. Intestinal stem cells lacking $C d c 42$ undergo defective cell division, abnormal morphogenesis, elevated apoptosis, and failed Paneth cell differentiation. Conversely, Rab8a regulates Cdc42-GTP activity in the intestinal epithelium but not in the mouse kidney, suggesting that the genetic interplay between these 2 small GTPases is essential and intensified during cell division, which is a primary feature of the small intestinal epithelium. Using imaging flow cytometry and live cell analyses, we demonstrate that inhibition of Cdc42 prevents cells from completing cytokinesis. Finally, mice haploinsufficient for both $C d c 42$ and Rab8a develop abnormal crypt morphology and show decreased epithelial nutrient uptake, an important physiological sign of MVID. These in vivo explorations of Cdc42-Rab8a interaction in intestinal crypt homeostasis and MVID pathogenesis provide genetic evidence for the involvement of stem cell defects in this digestive disease.

\section{Results}

Intestine-specific Cdc42 ablation disrupts epithelial morphogenesis. To investigate the contribution of $\mathrm{Cdc} 42$ to intestinal epithelial homeostasis, we first derived intestine-specific $C d c 42$-deficient mice (Cdc42 $2^{\text {loxP/loxP }}$;VillinCre, referred to as "mutant" henceforth). We used Western blot analysis to confirm an efficient Cdc42 ablation in intestines of mutant mice beginning on E15.5 (Figure 1A). $\mathrm{Pkc} \zeta$ and Par3 are 2 important components of Cdc42-Par-aPkc complex (14). No change was observed in total Pkç level, but phosphorylated $\mathrm{Pkc}^{\complement} \zeta\left(\mathrm{plkc}^{\complement} \zeta\right.$ ) was diminished in mutant intestines (Figure 1B), consistent with an abrogated Cdc42-Par-aPkc signaling pathway. In contrast, an increased Par3 protein level was detected in mutant intestines (Figure $1 \mathrm{~B}$ ). Cdc42 ${ }^{\text {loxP/loxP }}$; VillinCre mice started to appear smaller in size compared with their littermates from $\mathrm{P} 9$ and became severely growth-retarded after weaning (Supplemental Figure 1, A and B; supplemental material available online with this article; doi:10.1172/JCI60282DS1). The body weights of mutant mice plateaued around 3 months of age, until no feeding defect or reduced food intake was detected in comparison with the control mice. During this period, soft stools and anal swelling were frequently detected, but no intestinal bleeding was found in mutant mice. At 6 months of age, approximately $10 \%$ of the mutant mice died, with an average body weight of approximately one-third that of their control littermates (Supplemental Figure 1A), while no tumor was detected.
H\&E staining indicated a primary histological defect at the intervillus epithelial region in mutant embryonic intestines (Figure 1, C and D). At E16.5, a few days after VillinCre activation (15) and Cdc42 deletion (Figure 1A), when wild-type intervillus epithelial cells (Figure 1C) vigorously proliferate, mutant intervillus epithelial cells displayed abnormalities in cytoplasmic division and nuclear organization (Figure 1D). Postnatal mutant villus epithelial cells demonstrated a clear accumulation of vacuoles in their cytoplasm (indicated by arrows in Figure 1F). Substantial vacuolization was observed in post-weaning mutant intestinal epithelium and persisted throughout adulthood with increased severity (Supplemental Figure 1, C and D).

In sharp contrast to the presence of Paneth cells in control intestinal crypts, no typical Paneth cell granules were found in mutant crypts at any stage (lower panels in Supplemental Figure 1, C and D). Lysozyme staining revealed a clear mislocalization of lysozyme-positive cells to the mutant villi (arrows in Figure 1H). Control Paneth cells were found exclusively at the crypt bottom (Figure 1G). Some lysozyme-positive cells that remained in the mutant crypts exhibited a goblet cell-like morphology (Figure 1J), with increased cell volume compared with control Paneth cells (Figure 1I). Remarkably, transmission EM (TEM) analysis of postnatal and adult intestines revealed a complete absence of typical Paneth cell granules in 99\% of mutant crypts (status at 1 month shown in Figure 1, $\mathrm{K}$ and L). The EM morphology suggested that some mutant cryptic cells may possess goblet or enteroendocrine cell identify (Figure 1L). Indeed, Alcian blue and chromogranin A (ChgA) staining revealed significantly increased numbers of mucin-positive (Figure $1 \mathrm{~N}$ ) and ChgApositive enteroendocrine cells (Figure 1, Q and R) in mutant crypts, where a significant reduction of lysozyme-positive (Paneth) cells was found (Figure 1S).

Immunofluorescence analysis for alkaline phosphatase (AP), an enzyme exclusively targeted to the brush border in control enterocytes (Figure 1T), revealed cytoplasmic inclusions in mutant enterocytes (arrows in Figure 1U). Apical inclusion of AP was one of the diagnostic features for MVID (1). Mutant villus epithelium did elaborate the brush borders to some extent (Figure $1 \mathrm{U}$ ), and this was verified by a second brush border marker, villin (Supplemental Figure 1, E and F). In both fetal (Supplemental Figure 1, G and H) and adult intestines (Figure 1, V and W), E-cadherin and lectin DBA staining revealed dramatic disruptions of basolateral plasma membrane in mutant villus epithelia with frequent inclusions of lectin Dolichos biflorus agglutinin (DBA), an apically secreted glycoprotein, to the basolaterally located inter- or intracellular regions (arrows in Figure 1W and Supplemental Figure 1H). This basolateral defect was confirmed with two additional markers: the adhesion junction protein $\beta$-catenin and the ion transporter $\mathrm{Na}^{+}$/ $\mathrm{K}^{+}$-ATPase (Supplemental Figure 1, I and J). Furthermore, apical endosome clustering was disrupted in mutant cells (Supplemental Figure 1, K and L). Overall, intestine-specific Cdc42 deficiency affected intestinal crypt-villus epithelial morphogenesis.

Cdc42 deficiency affects stem cell division and clonal expansion. As Paneth cells have been recently identified as the niche signal providers for stem cells in the crypts (12), the crypt phenotypes of Cdc42-deficient mice prompted us to explore the underlying molecular changes that control crypt homeostasis in greater detail. Control crypts contained distinctively marked $\mathrm{Ki}^{+} 7^{+}$or $\mathrm{BrdU}^{+}$stem cells near the crypt bottom that were undergoing proliferation (arrows in Figure 2, A and C, and Supplemental Figure 2, A and C). In contrast, mutant progenitor cells appeared to be intermingled and became indistinguishable 

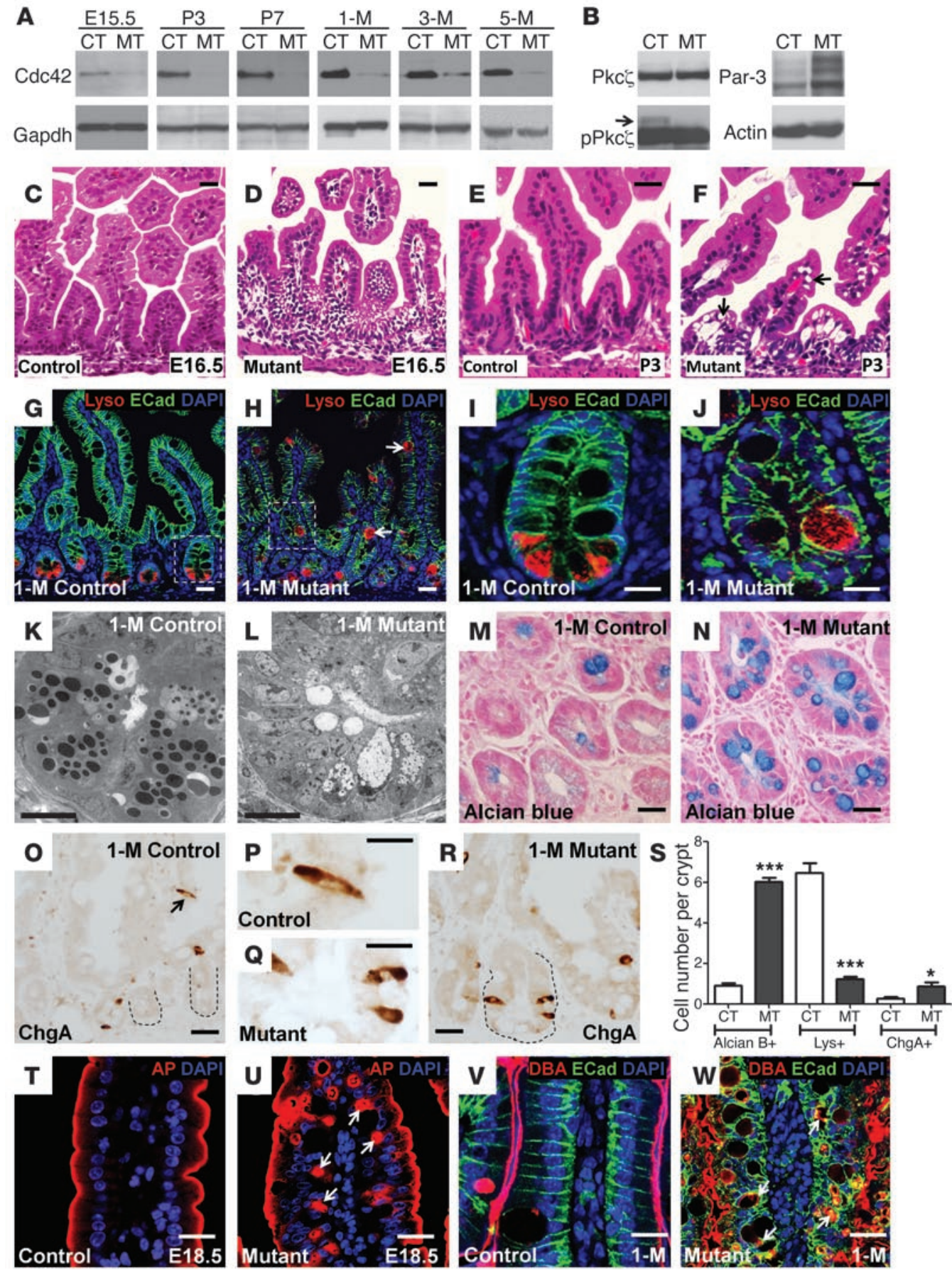

Figure 1

Cdc42 deficiency impairs intestinal epithelial morphogenesis. (A) Western blots for Cdc42 using control (CT) and mutant (MT) intestinal

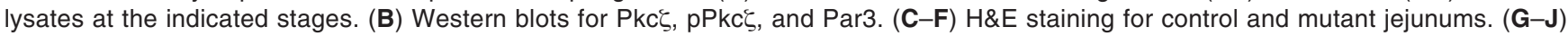
Immunofluorescence staining for lysozyme (red) and E-cadherin (green). Nuclei were labeled with DAPI (blue). Arrows in $\mathbf{H}$ indicate incorrectly localized lysozyme-positive cells in mutant villus epithelium. I and $\mathbf{J}$ show close-up images of the boxed regions in $\mathbf{G}$ and $\mathbf{H}$, respectively. (K and $\mathbf{L}$ ) TEM micrographs of 1-month-old (1-M) control and mutant jejunum crypts. ( $\mathbf{M}$ and $\mathbf{N}$ ) Alcian blue staining of 1-month-old control and mutant jejunum crypts. ( $\mathbf{O}-\mathbf{R})$ ChgA staining. Dotted lines in $\mathbf{O}$ and $\mathbf{R}$ indicate crypts. (S) Quantification of Alcian blue-positive (Alcian $\left.B^{+}\right)$, Lys ${ }^{+}$(lysozyme-positive), and $\mathrm{ChgA}^{+}$cell numbers per crypt. ( $\mathbf{T}$ and $\left.\mathbf{U}\right)$ Immunofluorescence staining for AP. Arrows in $\mathbf{U}$ indicate AP-positive inclusion bodies. ( $\mathbf{V}$ and $\mathbf{W}$ ) Immunofluorescence staining for DBA, lectin, and E-cadherin. Arrows in $\mathbf{W}$ indicate the vacuolar structures in mutant epithelial cells. ${ }^{*} P<0.05 ;{ }^{* *} P<0.001$. Data are mean \pm SEM. Scale bars: $10 \mu \mathrm{m}$ (K and $\left.\mathbf{L}\right), 2 \mu \mathrm{m}$ (all other panels). 

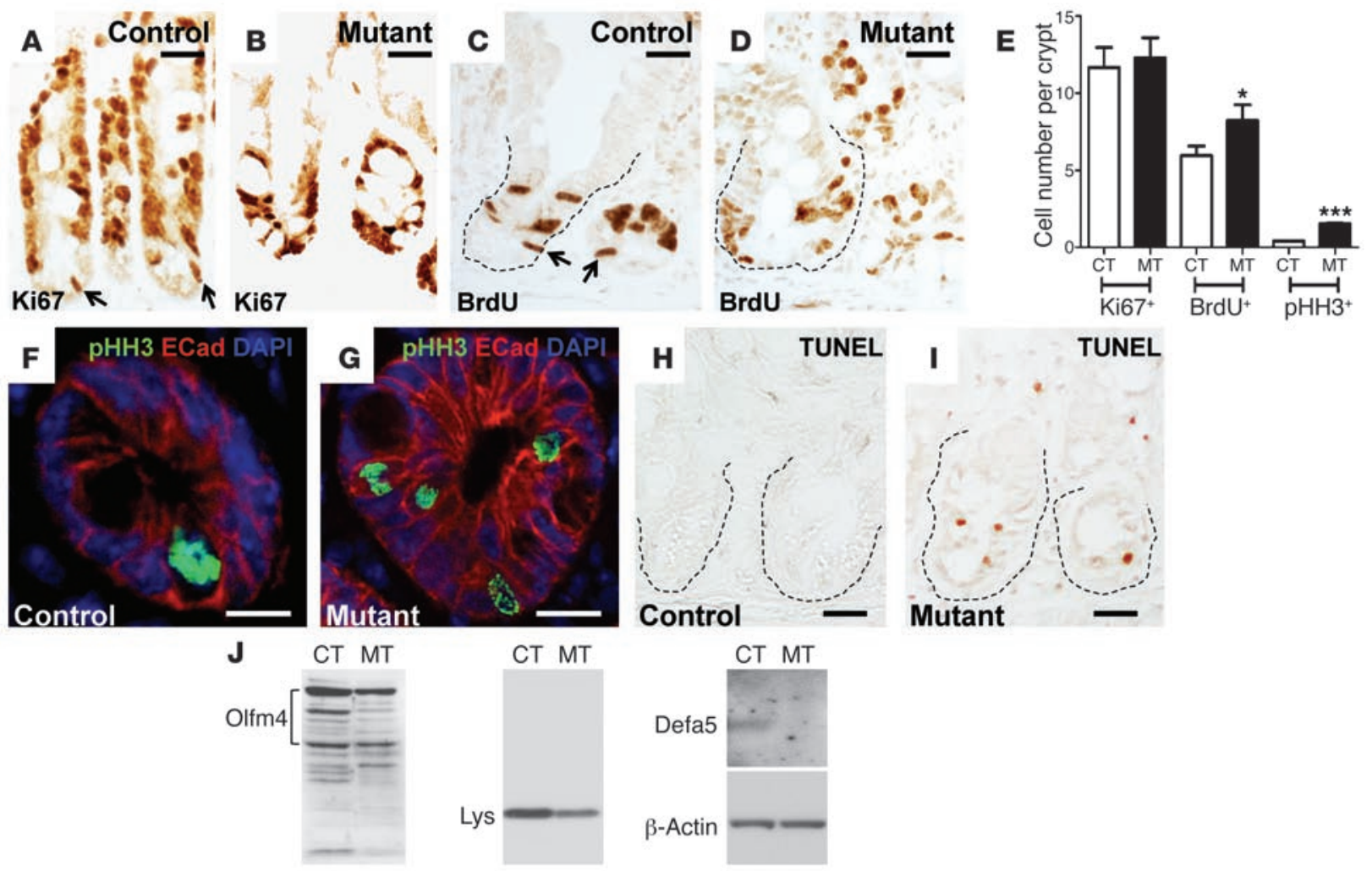

\section{Figure 2}

Cdc42 deficiency perturbs crypt homeostasis. (A and B) Ki67 staining of control and mutant intestinal crypts. Arrows in A indicate putative cycling stem cells. ( $\mathbf{C}$ and $\mathbf{D}$ ) BrdU staining. Arrows in $\mathbf{C}$ indicate putative stem cells at $\mathbf{S}$ phase. Dotted lines in $\mathbf{C}$ and $\mathbf{D}$ indicate crypts. (E) Quantification of $\mathrm{Ki}^{+} 7^{+}$, BrdU ${ }^{+}$, and $\mathrm{pHH} 3^{+}$cells per crypt. ${ }^{*} P<0.05$; ${ }^{* * *} P<0.001$. ( $\mathbf{F}$ and $\mathbf{G}$ ) Immunofluorescence staining of pHH3 and E-cadherin. ( $\mathbf{H}$ and $\left.\mathbf{I}\right)$ TUNEL staining. Crypts are indicated by dotted lines. (J) Western blots for Olfm4 and lysozyme in total intestinal lysates. AU-PAGE analysis for Defa5. $\beta$-Actin served as a loading control. Scale bars: $5 \mu \mathrm{m}$.

from transit amplifying cells (Figure 2, B and D, and Supplemental Figure 2, B and D). Although no difference was detected between control and mutant crypts for the total cycling cells per crypt, 6-hour pulse BrdU labeling revealed a slightly increased BrdU ${ }^{+}$cell number in mutant crypts (Figure 2E). An increased number of mutant cells undergoing mitosis was also revealed by $\mathrm{pHH} 3$ staining (Figure 2, E-G), indicating that $C d c 42$ deficiency either triggered cell proliferation or blocked cell cycle exit. Strikingly, TUNEL analyses revealed a dramatically increased apoptotic cell number in Cdc42-deficient crypts, with TUNEL ${ }^{+}$cells detected in the majority of mutant crypts (Figure 2I and Supplemental Figure 3, B and D), suggesting that cell division defects might have triggered increased cell deaths. In contrast, apoptosis was only found at villus tips but not crypts in control intestines (Figure 2H and Supplemental Figure 3, A and C).

Western blots revealed decreased protein levels of Olfm 4 and lysozyme, markers for stem cells and Paneth cells, respectively (Figure 2J), indicating decreased stem and Paneth cell populations in mutant crypts. Acetic acid-urea polyacrylamide gel electrophoresis analysis further confirmed the loss of a Paneth cell-specific defensin (Defa5) in the mutant intestines (Figure 2J). These data suggested that $C d c 42$ deficiency triggered cell cycle defects that lead to increased apoptosis.

The VillinCre deletes gene targets in all intestinal epithelial cell types including the stem cell population (15). In order to achieve stem cell-specific $C d c 42$ ablation and lineage tracing of $C d c 42$ - deficient stem cells, we employed an inducible gene ablation with $\operatorname{Lgr} 5^{C r e E R-E G F P}$ mice (16). Prior to tamoxifen administration, $C d c 42^{\operatorname{loxP} / \operatorname{loxP}} ; \operatorname{Lgr} 5^{\text {CreER-EGFP }}$ mouse crypts demonstrated interspersed stem cell and Paneth cell organization indicative of normal crypt homeostasis (Figure 3, A and B). The mosaicism of Lgr5CreER-EGFP seen in our experiments was consistent with a previous report (16). One week after tamoxifen administration (Figure 3C), stem cells in $56 \%$ of $\mathrm{GFP}^{+} \mathrm{Cdc} 42^{\text {loxP } / \text { loxP }} ; \operatorname{Lgr} 5^{\text {CreER-EGFP }}$ mouse crypts began forming a "clustering" pattern lacking clear demarcation between each other (arrows in Figure 3E), while the stem cells in over 90\% of $\mathrm{GFP}^{+}$(i.e., genetically labeled) $\operatorname{Lgr} 5^{\mathrm{Cr} E E R-E G F P}$ control crypts were separated from the neighboring stem cells by Paneth cells (Figure 3D). We defined this stem cell clustering phenotype as four or more $\mathrm{GFP}^{+}$cells aggregated together in the crypt, and we quantified the frequency of such clustering in mice that had been treated with tamoxifen 1 and 3 weeks previously (Figure 3L). Three weeks after tamoxifen administration, $\mathrm{GFP}^{+} \mathrm{Cdc} 42^{\text {loxP/loxP }} ; \operatorname{Lgr} 5^{\text {CreER-EGFP }}$ crypts that contained the clustered stem cells increased to $85 \%$, while no significant change was seen in $\mathrm{GFP}^{+} \operatorname{Lgr} 5$ CreER-EGFP control mouse crypts (Figure 3, F, G, and L). In addition, stem cells in $C d c 42^{\text {loxP/loxP }} ; \operatorname{Lgr} 5^{\text {CreER-EGFP }}$ crypts 3 weeks after tamoxifen treatment (short arrows in Figure 3, J and N) lost the typical triangle shapes seen in the stem cells of control crypts (Figure 3, D, F, H, and $\mathrm{M}$ ). As their morphologies changed, a simultaneous loss of apical Pkc' $\zeta$ in these $C d c 42^{\operatorname{loxP} / \operatorname{lox} P} ; \operatorname{Lgr} 5^{\text {CreER-EGFP }}$ crypts was detected 

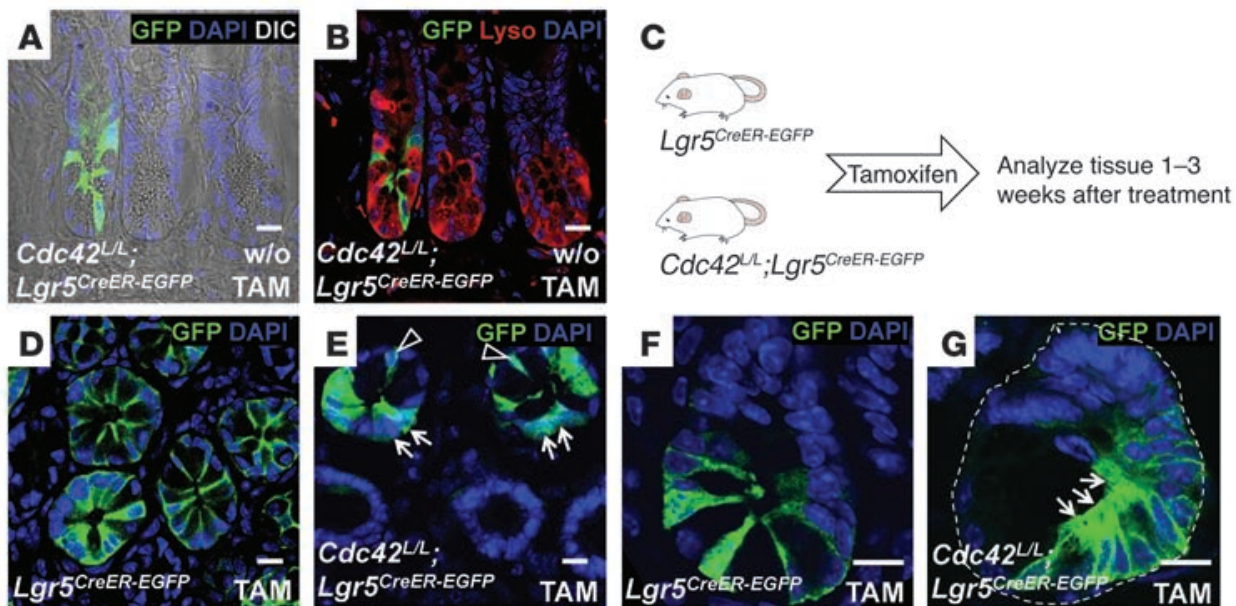

Cdc $42^{\Omega}$;Lgr5 CrEER-EGFP
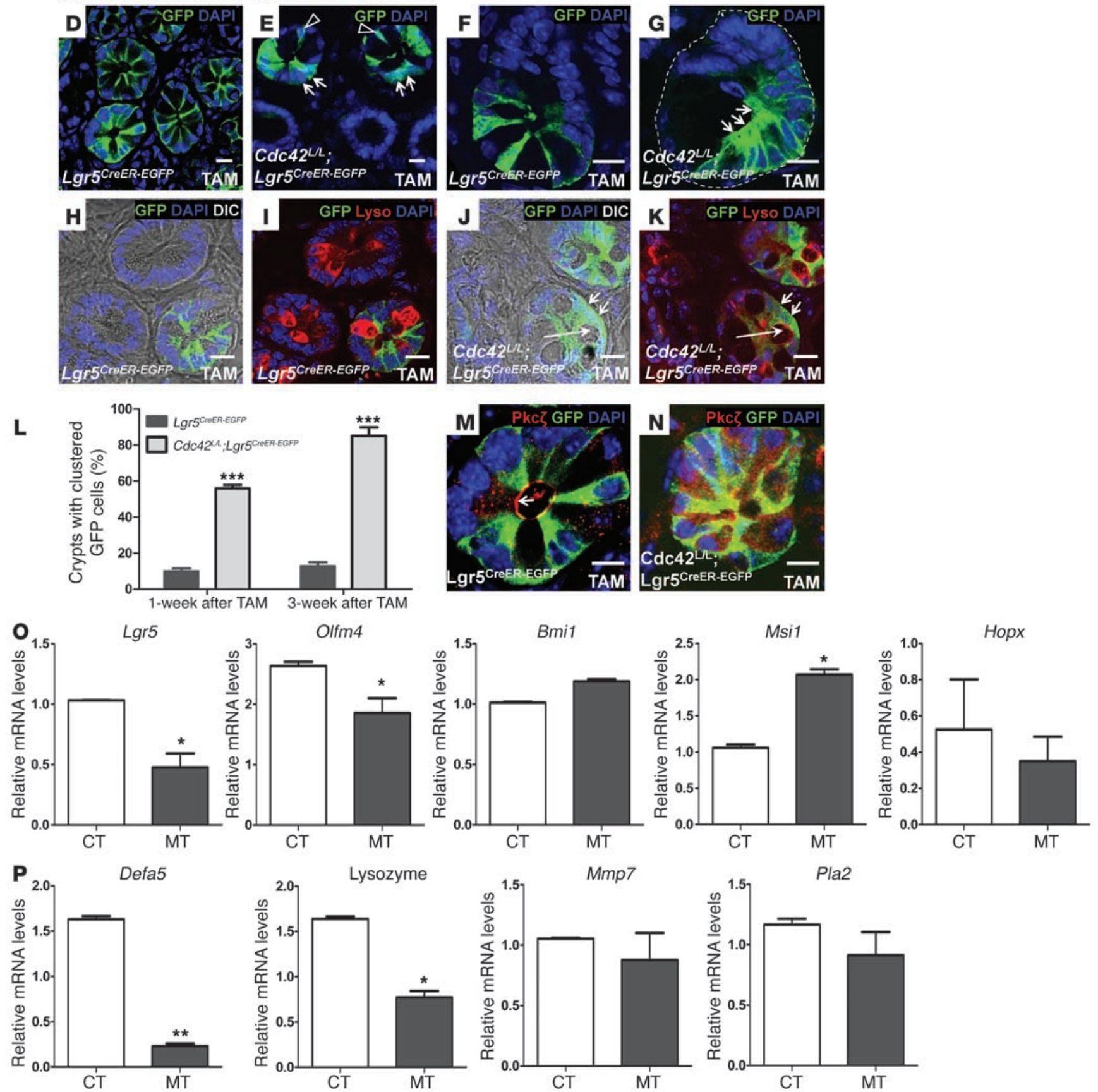


\section{Figure 3}

Defective cell division and differentiation of Cdc42-deficient Lgr5 stem cells. (A and B) Immunofluorescence staining for lysozyme and GFP in Cdc42 ${ }^{L / L}$;Lgr5CreER-EGFP mouse crypts prior to tamoxifen treatment. Paneth cell granules are visible in $\mathbf{A}$. L/L indicates LoxP/loxP. (C) Experimental schematic of tamoxifen-induced $\mathrm{Cdc42}$ ablation in Lgr5 stem cells. (D and E) GFP staining of control and Cdc42-deficient intestinal crypts 1 week after tamoxifen administration. Arrows in $\mathbf{E}$ indicate clustered stem cells; arrowheads point to stem cells that are segregated from neighboring stem cells. (F and $\mathbf{G}$ ) GFP staining 3 weeks after tamoxifen treatment. Clustered stem cells are indicated by arrows in G. Dotted line in $\mathbf{G}$ indicates a crypt. (H-K) GFP and lysozyme staining for control and Cdc42-deficient intestinal crypts 3 weeks after tamoxifen administration. Short arrows in $\mathbf{J}$ indicate clustered stem cells losing their triangle shape. Long arrow points a large vacuole in the mutant crypt. Paneth cell granules are visible in control crypts in $\mathbf{H}$, but not in Cdc42-deficient crypts in $\mathbf{J}$. (L) Cdc42-deficient crypts show increased frequency of abnormal clustering of GFP+ Lgr5 stem cells. ( $\mathbf{M}$ and $\mathbf{N}$ ) Costaining for $\mathrm{Pkc} \zeta$ and GFP. Arrow in $\mathbf{M}$ points to apical Pkc $\zeta$ staining. ( $\mathbf{O}$ and $\mathbf{P})$ Real-time RT-PCR analyses. Scale bars: $5 \mu \mathrm{m} .{ }^{*} P<0.05 ;{ }^{* *} P<0.01$; ${ }^{* *} P<0.001$.

(Figure 3, M and N), confirming a disrupted crypt cell polarity. Using differential interference contrast (DIC) and lysozyme staining, we found a clear reduction or absence of Paneth cell granules and an appearance of cells containing large vacuoles in $\mathrm{GFP}^{+}$ $C d c 42^{\text {loxP/loxP }} ; \operatorname{Lgr}^{\text {CreER-EGFP }}$ crypts (long arrows in Figure 3, J and $\mathrm{K}$ ) compared with control $\mathrm{GFP}^{+}$crypts (Figure $3, \mathrm{H}$ and I). Because the lysozyme antibody weakly stained these vacuolar structures (Figure 3K), we suspected that these were Paneth cells that existed before the tamoxifen treatment.

Quantitative RT-PCR revealed significantly reduced stem cell (Lgr5 and Olfm4) and Paneth cell markers (Defa5 and Lysozyme) (Figure 3, $\mathrm{O}$ and $\mathrm{P}$ ), supporting a loss of these cells in the mutant crypts (Figure 2I and Figure 3J). Interestingly, we did not detect a significant decrease in other stem cell (Bmi1, Msi1, or Hopx) or Paneth cell (Mmp7 or Pla2) markers (Figure 3, O and $\mathrm{P}$ ), indicating that a compensation for the loss of Lgr5 stem cells by non-Lgr5 slow cycling stem cells may be at play $(17,18)$.

To test the clonal expansion and differentiation capacities of $\mathrm{Cdc42-}$ deficient $\mathrm{Lgr} 5^{+}$stem cells, we performed lineage-tracing experiments using Rosa-YFP mice (Figure 4A). Two weeks after tamoxifen administration, within genetically labeled $\mathrm{GFP}^{+} / \mathrm{YFP}^{+} \operatorname{Lgr} 5^{\text {CreER-EGFP }}$;RosaYFP control crypts, all cells were stained positively by a GFP antibody that recognized both stem cells $\left(\mathrm{GFP}^{+}\right)$and their descendents $\left(\mathrm{YFP}^{+}\right)$ (Figure 4B). Cells that were positive for both GFP/YFP and lysozyme were detected in control crypts (Figure 4B), indicating normal differentiation of $\mathrm{Lgr}^{+}{ }^{+}$stem cell into Paneth cells (16). In contrast, Cdc42deficient stem cells in $C d c 42^{\text {loxP/loxP }} ; \operatorname{Lg} 5^{\text {CreER-EGFP }}$;RosaYFP intestinal crypts failed to give rise to Paneth cells, as indicated by a lack of colocalization of GFP/YFP- and lysozyme-positive signals in at least $90 \%$ of GFP/YFP-labeled crypts (Figure 4C).

Three weeks after tamoxifen administration we observed green stripes of $\mathrm{GFP}^{+} / \mathrm{YFP}^{+}$villi in $\operatorname{Lgr} 5^{\mathrm{CreER}-E G F P}$;RosaYFP control intestines that were always accompanied by positively-labeled $\left(\mathrm{GFP}^{+}\right)$ crypts at the bottom (Figure 4D). Due to the mosaicism of Lgr $5^{\text {CreER-EGFP }}$ mouse line, we also frequently found $\mathrm{GFP}^{-} / \mathrm{YFP}^{-}$villi. Unexpectedly, this mosaic activity of $\operatorname{Lgr} 5^{\text {CreER-EGFP }}$ provided us an opportunity to compare the clonal expansion capacities between Cdc42-deficient stem cells and "wild-type" stem cells (i.e., GFP-/ YFP- cells that escaped Cre recombination) within the same tamoxi-

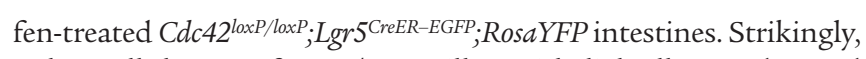
only small clusters of $\mathrm{GFP}^{+} / \mathrm{YFP}^{+}$villus epithelial cells were detected in $C d c 42^{\text {loxP/loxP }} ; \operatorname{Lgr} 5^{\text {CreER-EGFP }} ;$ RosaYFP mice 3 weeks after tamoxifen injection (Figure 4E). Within the positively labeled villi of $C d c 42^{\text {loxP } / l o x P}$;

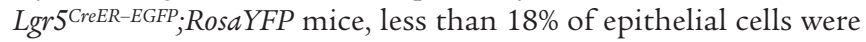
labeled by GFP/YFP. In contrast, $92 \%$ of epithelial cells within the positively labeled (i.e., $\mathrm{GFP}^{+} / \mathrm{YFP}^{+}$) Lgr $5^{\mathrm{CreER}-E G F P}$;RosaYFP villi were found to be labeled green (Figure 4F). These data suggested a reduced clonal expansion capacity of $\mathrm{Cdc} 42$-deficient stem cells compared with their wild-type counterparts. Careful examination of these small clusters of $\mathrm{GFP}^{+} / \mathrm{YFP}^{+}$villus epithelial cells in $C d c 42^{\operatorname{loxP} / \operatorname{lox} P} ; \operatorname{Lgr} 5^{\mathrm{CreER}-E G F P} ;$ RosaYFP mice revealed a disrupted cell polarity, as indicated by disorganized nuclear alignment (yellow arrowheads in Figure 4, $\mathrm{K}$ and $\mathrm{L}$ ). In contrast, $\mathrm{GFP}^{+} / \mathrm{YFP}^{+}$villus cells

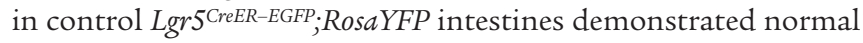
columnar shapes with their nuclei well-aligned against the basement membrane (arrowheads in Figure 4, H and I). Remarkably, within the same $C d c 42^{\text {loxP/loxP }} ; \operatorname{Lgr} 5^{\text {CreER-EGFP }}$ RosaYFP intestinal villi, neighboring epithelial cells that were $\mathrm{GFP}^{-} / \mathrm{YFP}^{-}$(i.e., wild-type stem cell descendents) showed normal morphology (compare the white arrowheads with yellow arrowheads in Figure 4L).

Furthermore, costaining of $\mathrm{pHH} 3$ and GFP in $\operatorname{Lgr}^{\text {CreER-EGFP; }}$ RosaYFP and Cdc42 $2^{\operatorname{loxP} / \operatorname{lox} P} ; \operatorname{Lgr} 5^{\mathrm{CreER}-E G F P} ;$ RosaYFP intestinal crypts from tamoxifen-treated animals confirmed that more $C d c 42$-deficient $\mathrm{Lgr}^{+}$stem cells $\left(\mathrm{GFP}^{+}\right.$crypt in Figure $\left.4 \mathrm{~N}\right)$ were undergoing mitosis compared with Lgr $5^{\text {CreER-EGFP }}$;RosaYFP wild-type stem cells (Figure 4M) and compared with the wild-type stem cells in $C d c 42^{\text {loxP/loxP }}$; Lgr 5 CreER-EGFP; RosaYFP crypts (circled GFP- crypts in Figure 4N). Thus, Cdc42-deficient Lgr5 stem cells contributed less to the villus epithelial compartments (Figure 4, E and F), had abnormal morphology (Figure 3, G, J, and N), and showed increased cell death (Figure 2I). These data indicate that the reduced clonal expansion of $C d c 42$-deficient stem cells may result from a defect causing slow mitotic progression or mitotic arrest.

Cdc42 deficiency induces MVID. While we were searching for the molecular mechanism underlying the stem cell division defects, TEM analysis of postnatal and adult $C d c 42^{\text {loxP/loxP }}$; VillinCre mutant mouse intestines revealed a dramatic microvillus inclusion phenotype that was reminiscent of Rab8a-knockout mouse intestine and human intestinal MVID (4). Numerous microvillus inclusion bodies (vacuoles) were found in mutant enterocytes residing in both upper (arrows in Figure 5B) and lower villus epithelial regions (arrows in Figure 5C). This was consistent with the abnormal AP staining in mutant epithelium (Figure 1U). High-magnification EM imaging revealed the presence of microvilli at the inner surfaces of these inclusion vacuoles (Figure 5D). In contrast to control enterocytes that never exhibited any microvillus inclusion (Figure 5A), mutant enterocytes demonstrated small inclusion bodies as early as P7 (arrows in Figure 5, E and F), with typical actin filaments bundled at the core of included microvilli (Figure 5G). These inclusion bodies failed to disappear, and instead became further enlarged in aged mutant mouse enterocytes (status at 3 months is shown in Figure $5 \mathrm{H}$ ). We found a rare incidence of microvillus inclusion body within the tamoxifen-treated $C d c 42^{\text {loxP } / \text { loxp }} ; \operatorname{Lgr} 5^{\text {CreER-EGFP }}$ mouse intestines, suggesting that the $C d c 42$-deficient stem cells were prevented by programmed cell death from contributing to the abnormal epithelial structure (Figure 2I).

Cdc42 controls Rab8a vesicle traffic and Rab8a-GTP activity. Due to the phenotypic similarity between $C d c 42$ - and Rab8a-knockout mouse intestines, we examined the total Rab8a protein levels in 

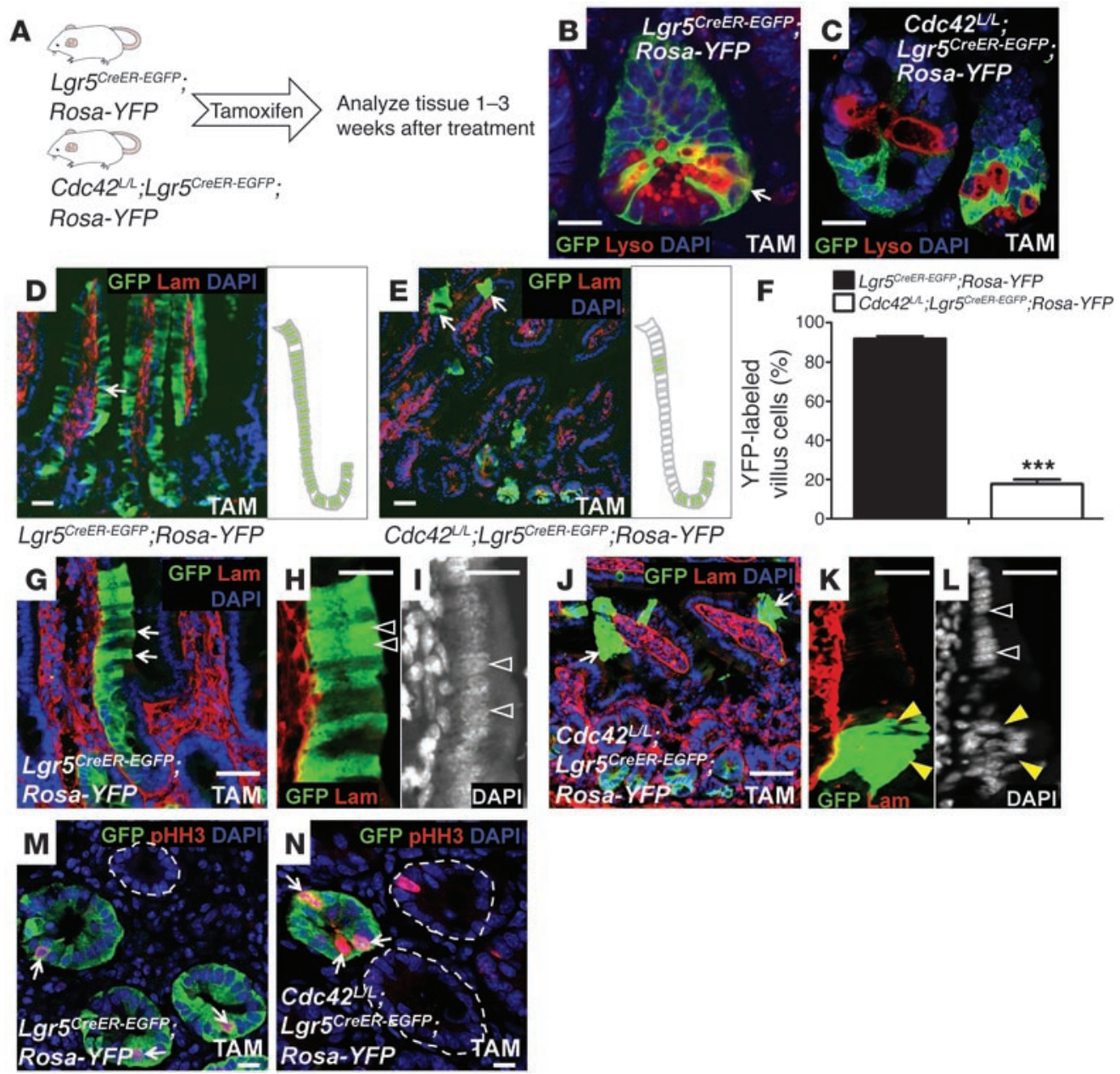

\section{Figure 4}

Cdc42 deficiency reduces the clonal expansion capacity of Lgr5 stem cells. (A) Experimental scheme for the genetic tracing experiments. (B and C) GFP and lysozyme staining for control and Cdc42-deficient crypts 2 weeks after tamoxifen treatment. Arrow in B points to cells (in yellow) positive for both GFP and lysozyme. (D and E) GFP and basement membrane staining for control and Cdc42-deficient crypts 3 weeks after tamoxifen treatment. Arrows in E point to small clusters of cells derived from Cdc42-deficient stem cells. Schematic diagrams at the right of each panel summarize the results shown at left. (F) Quantification of RosaYFP-labeled villus epithelial cells derived from control and Cdc42-deficient Lgr5 stem cells 3 weeks after tamoxifen administration. ${ }^{* *} P<0.001$. (G-L) GFP and basement membrane staining illustrate columnar shapes of labeled control intestinal epithelial cells (arrows in G) but abnormal morphology of labeled Cdc42-deficient cells (arrows in J). Arrowheads in $\mathbf{H}$ and $\mathbf{K}$ indicate positively labeled villus epithelial cells. White arrowheads in $\mathbf{I}$ and $\mathbf{L}$ indicate normal nuclear alignment. Yellow arrowheads in $\mathbf{L}$ indicate disrupted nuclear organization and cell polarity in villus cells derived from Cdc42-deficient stem cells. (M and $\mathbf{N}$ ) Costaining of pHH3 and GFP. Dotted line in $\mathbf{M}$ indicates an unlabeled crypt. Dotted lines in $\mathbf{N}$ encircle the wild-type crypts that escaped Cre recombination. Arrows in $\mathbf{M}$ and $\mathbf{N}$ indicate GFP+/pHH3 ${ }^{+}$cells. Scale bars: $5 \mu \mathrm{m}$.

control and $C d c 42^{\text {loxP/loxP }}$; VillinCre mutant intestines using Western blot analysis. Protein levels of Rab8a and its critical regulators, Rab11a and Rabin8 $(11,19)$, did not change at the embryonic or adult stages examined (Figure 6A). To test whether the levels of activated GTP-bound Rab8a were altered in mutant intestines, we employed a pull-down assay using GST-JFC1 fusion protein as the bait (Figure 6B). JFC1 is a synaptotagmin-like protein that binds to activated Rab8a-GTP rather than to Rab8a-GDP (20). Mutant intestines consistently demonstrated $85 \%-90 \%$ reduction in Rab8aGTP level compared to control intestines at both juvenile or adult stages (Figure 6B). In addition to reduced Rab8a-JFC1 interaction, mutant intestinal epithelium also demonstrated a decreased association between Rab8a and its downstream effector, the exocyst (ref. 21 and Figure 6C).
A recent study has proposed that Rab proteins regulate global Cdc42 activation in the MDCK cell line (11). We examined Cdc42GTP levels in 3-week-old Rab8a-knockout mouse intestines and indeed identified a reduction of total and activated Cdc42-GTP levels (Figure 6D). This reduced Cdc42 activation was consistent with a diminished pPkc $\zeta$ level in $R a b 8 a^{-/-}$mouse intestines (Figure 6D). To explore whether this regulation also occurred in Rab8a-knockout kidney, in which no phenotype was identified, we measured the Cdc42GTP levels and detected no reduction (Supplemental Figure 4). This result to some extent explained the observation of normal epithelial polarity and structure in Rab8a-knockout kidneys (4) and further indicated that the regulation between Cdc42 and Rab8a appeared to depend on or intensify during active cell division, which was the primary feature of the small intestinal epithelium. 

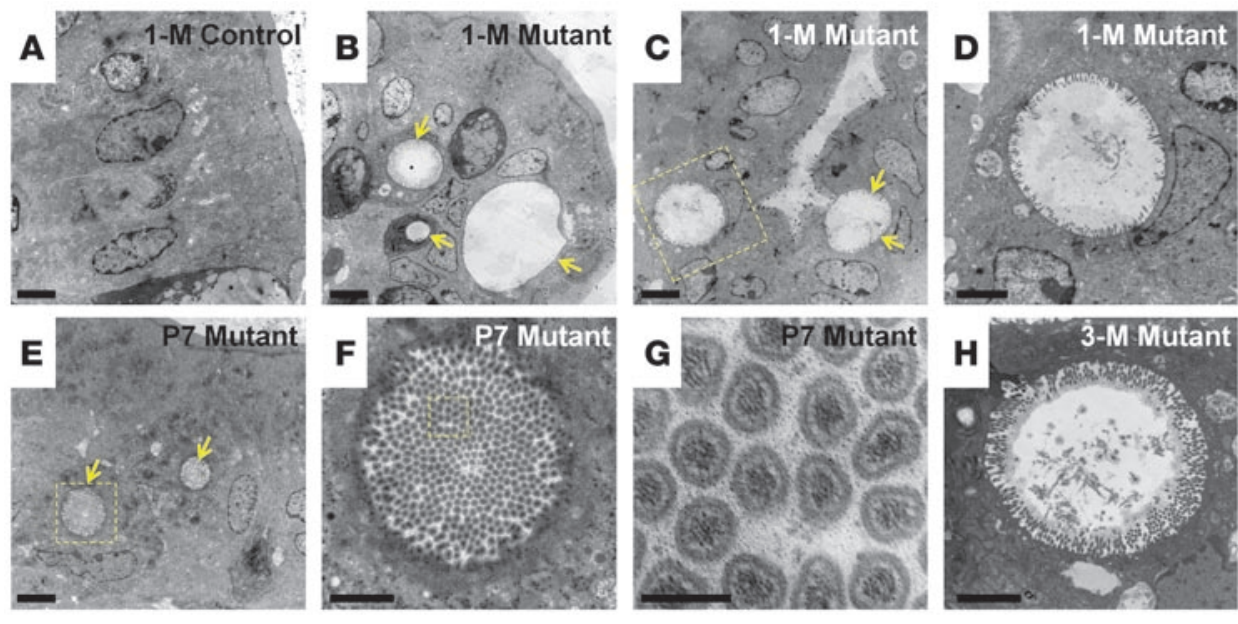

\begin{abstract}
Figure 5
Cdc42-deficient enterocytes develop microvillus inclusions. TEM micrographs of control and Cdc42-mutant intestines at the indicated ages. Arrows in $\mathbf{B}, \mathbf{C}$, and $\mathbf{E}$ indicate microvillus inclusion bodies. Scale bars: $2 \mu \mathrm{m}(\mathbf{A}-\mathbf{C}$ and $\mathbf{E}), 500 \mathrm{~nm}$ (D, F, and H), $100 \mathrm{~nm}(\mathbf{G})$.
\end{abstract}

Since diminished Rab8a activation was accompanied by no detectable change in protein levels of the Rab8a activators, Rab11a and Rabin8 (Figure 6A), we suspected that Rab8a vesicle traffic may be dependent on $\mathrm{Cdc} 42$ during cell division. We employed a previously described SMARTpool of siRNAs against $C d c 42(22)$ that resulted in knockdown of over $90 \%$ of Cdc42 in Hela cells (Figure 7A). Cdc42 knockdown did not affect Rab8a localization around the microtubule organization center in cells at interphase (Supplemental Figure 5, $\mathrm{A}$ and $\mathrm{B})$. In contrast, $\mathrm{Cdc} 42$ depletion reduced the traffic of Rab8aGFP to the midbody during cytokinesis in either live cells expressing mcherry-tubulin (Figure 7C) or in fixed cells stained for tubulin (Figure 7E). Effective recruitment of Rab8a-GFP to the midbody was detected in control cells (arrows in Figure 7B). Similar observations were made in cells at late cytokinesis (Figure 7, D and E), and the difference was significant (Supplemental Figure 5C). Although cells progressing at various mitotic phases were still detected upon $\mathrm{Cdc} 42$ knockdown (Supplemental Figure 5D), we observed an approximately 3-fold reduction in cytokinetic events in Cdc42-depleted cells compared with control knockdown cells (Figure 7F and Supplemental Figure 5D). Treatment of cells with $7 \mu \mathrm{M}$ CASIN, a Cdc42 chemical inhibitor (23), prevented cytokinesis to a greater extent (Figure 7F). An elevated dosage $(20 \mu \mathrm{M})$ of CASIN killed all cells within 24 hours (data not shown). FACS analyses revealed a 19\% accumulation (i.e., from $9.7 \%$ to $11.5 \%$ ) of cells at the $\mathrm{G}_{2} / \mathrm{M}$ phase after $7 \mu \mathrm{M}$ CASIN treatment (Figure 7G and Supplemental Figure 6). Furthermore, imaging flow cytometry confirmed that this increase was primarily contributed by cells at late mitosis rather than at the $\mathrm{G}_{2}$ phase, as $7 \mu \mathrm{M}$ CASIN treatment dramatically enriched cells with anaphase morphology (Supplemental Figure 7).

Cdc42-regulated Rab8a traffic is coupled with proper polarity formation. To test whether Cdc42-controlled Rab8a vesicle traffic is essential for coupling cytokinesis to polarity establishment, we employed the 3D Caco 2 cystogenesis model after depleting $\mathrm{Cdc} 42$ by lentiviral shRNA particles (Figure $7 \mathrm{H}$ ). Control knockdown cells correctly oriented the mitotic spindle axis parallel to the apical surface and positioned the midbody toward the cystic center (white arrowhead in Supplemental Figure 8A), consistent with a previous report (22). In contrast, $C d c 42$ depletion disrupted spindle and midbody orientations (Supplemental Figure 8B).

Before open lumen formation, Rab8a vesicles clustered to the prospective apical domain (Supplemental Figure 8C), where the midbody was positioned (Supplemental Figure 8A). After lumen formation these vesicles primarily targeted to the sub-apical regions where the $\mathrm{Cdc} 42$ effector $\mathrm{Pkc} \zeta$ was localized (Figure 7I). In contrast, Cdc42 depletion induced a significantly misoriented traffic of Rab8a vesicles during early (Supplemental Figure 8D) and late cystogenesis (Figure 7J). Interestingly, DIC images of Cdc42-knockdown cysts illustrated a severe vacuolar accumulation at the basolateral region, where Rab8a was detected (arrowheads in Figure 7J). Furthermore, in the absence of Cdc42, Rab8a vesicles were mistargeted to $\mathrm{Pkc}^{\mathrm{C}} \zeta^{+}$sites, where ectopic lumens were formed (Figure 7J). These in vitro cystogenesis data were consistent with the Cdc42deficient intestinal epithelial phenotype (Figure $1 \mathrm{~W}$ ), which strongly suggests that $\mathrm{Cdc} 42$ controls Rab8a vesicle traffic for midbody positioning, cytokinesis, and epithelial morphogenesis.

Genetic association between Cdc42 and Rab8a. Genetic interaction between Cdc42- and Rab8a-regulated molecular machineries has never been tested an in vivo mammalian system. The dramatic MVID phenotypes observed in both mouse models indicated a functional crosstalk between these 2 small GTPases during gut epithelial development. We then derived $\mathrm{Cdc} 42^{\text {loxP/+}} ;$ Rab8 $8 a^{+/-}$; VillinCre (referred to hereafter as "double heterozygous") mice that were haploinsufficient for both genes in the intestinal epithelium. TEM analysis revealed greatly expanded (Figure $8 \mathrm{~B}$ ) or elongated (Figure 8C) crypts with large lumens and vacuoles in some double heterozygous intestinal crypts, compared with control (Figure 8A) or single heterozygous crypts (Supplemental Figure 9A). The inner surfaces of these cryptic lumina/vacuoles were lined by microvilli (Figure 8D and Supplemental Figure 9A). Although Paneth cell granules were present in the crypts of double heterozygous mice (Figure 8, B and C), the electron intensities of Paneth cell granules were markedly reduced in comparison with control granules (Figure $8 \mathrm{~A}$ ). To test the physiological functions of single and double heterozygous mouse intestines, we performed in vivo epithelial uptake assays using radioactively labeled glucose, carnosine, and proline. In comparison with Cdc42 and Rab8a single heterozygous mice, double heterozygous mice showed a significant reduction in glucose uptake (Figure 8E), suggesting an additive effect. Cdc42deficient intestines demonstrated significantly reduced nutrient uptake activities for all 3 substances with almost no absorption of proline (Figure 8, E-G). This decreased glucose transporter activity (Figure 8E) was in agreement with a reduced level of apically targeted sodium/glucose cotransporter member 1 (Sglt1), in Cdc42-deficient and double heterozygous intestines (Supple- 
A
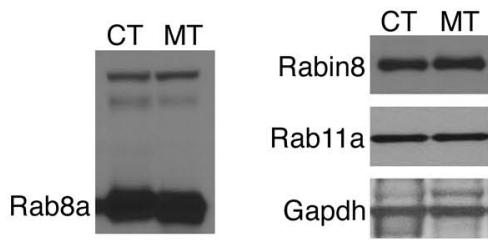

C

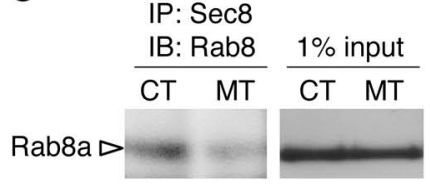

B
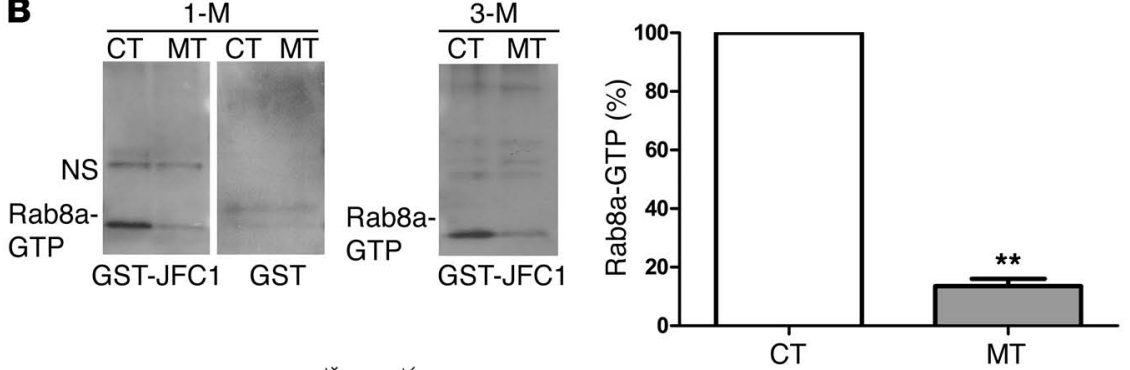

Figure 6

Cdc42 deficiency impairs Rab8a activation. (A) Western blots for Rab8a, Rab11a, and Rabin8. (B) GST-JFC1 pull-down assay followed by Western blots for Rab8a showed significantly lower Rab8a-GTP levels in 1-month-old and 3-month-old mutant intestines. NS, non-specific bands. ${ }^{\star *} P<0.01$. (C) Co-IP for Sec8 and Rab8a demonstrated reduced association of Rab8a with exocyst. (D) In 3-week-old Rab8-- intestine, total Cdc42, Cdc42GTP, and pPkç levels were reduced compared with control intestine. Western blot results represent at least 3 independent experiments.

mental Figure 9, B and C). Finally, double heterozygous mice and Cdc42-deficient mice both demonstrated increased intestinal tissue weight per surface area (Supplemental Figure 10A), indicating a degree of tissue edema that would be consistent with the increased epithelial apoptosis (Figure 2I) since no significant increase was detected in the average dimensions of mutant intestinal crypt and villi (Supplemental Figure 10, B-F).

\section{Discussion}

In this study we employed multiple genetic mouse models and identified the essential contribution of $\mathrm{Cdc} 42$ to the small intestinal stem cell division and epithelial homeostasis. We explored the molecular ontogenesis of MVID in a new mouse model that recapitulates the disease at EM and physiological levels. Using both in vivo and in vitro methods, we demonstrated the genetic interplay between Cdc42 and Rab8a, 2 well-conserved prototypic small GTPases of the Rho and Rab subfamily, respectively. We propose that the constant cell division of the small intestinal stem cells is dependent on the coordination of Cdc42 and Rab8a, and that the primary location of their functional interaction happens at the midbody, a trafficking hub that plays a fundamental role in cytokinesis and subsequent polarity determination. Most importantly, by investigating the stem cell compartment in MVID disease models, our study represents a conceptual departure from the conventional view of this disease as occurring at the abnormal enterocytes.

In comparison with its role in epithelial polarity, the involvement of Cdc42 in cytokinesis has been relatively less explored. Using live cell imaging and imaging flow cytometry, we demonstrate the blockage of cytokinesis in Cdc42-knockdown or CASIN-inhibited cells. These in vitro findings were consistent with the defective stem cell division and elevated crypt apoptosis observed in Cdc42deficient intestines. Our data revealed the molecular mechanism that potentially contributes to the blocked cytokinesis: that is, the Cdc42-dependent midbody trafficking of Rab8a vesicles. Indeed, in fission yeast, $\mathrm{Cdc} 42$ preferentially localizes to the division site and has been suspected to recruit molecular components for the contraction of actomyosin ring (24). Dominant-negative or constitutively active Cdc42 appears to block the division of Xenopus embryos (25). Further, toxin B treatment of mouse oocytes and cleaving embryos with toxin B, which presumably inhibits Cdc 42 binding, also affected the cytokinesis of the treated embryos (26). Most importantly, our multiple loss-of-function genetic experiments in mice not only affirmed the role of $\mathrm{Cdc} 42$, but provide further genetic evidence linking Cdc42- and Rab8a-mediated signaling and trafficking pathways.

With the recently available genetic tools (16), our tracing experiments identified that $\mathrm{Cdc} 42$ was indispensible for the clonal expansion capacity of Lgr5 intestinal stem cells, which represent the fast-cycling stem cells in the crypts. The overwhelming microvillus inclusion phenotype observed in Cdc42-VillinCre mouse intestines was not surprising because comprehensive deletion of $C d c 42$ in the entire epithelium greatly blocked the potential compensation from wild-type stem cells. The rare incidence of MVID in Cdc42-Lgr 5 CreER mutant mice further illustrated the powerful surveillance and compensatory mechanism employed by the epithelial crypts to limit the capacity of abnormal stem cells for contributing to their epithelial descendent cells. Most importantly, we were the first to demonstrate that in the same crypts, wild-type stem cells outcompeted the Cdc42deficient stem cells that had impaired expansion capacity. In our view, this observation provides promise for the intervention of this disease with regenerative medicine via replacement of the diseased tissues with healthy stem cells or crypts. Our results also advocate for searching for additional genetic or epigenetic variations that may potentially affect stem cell division in MVID patients. 
A

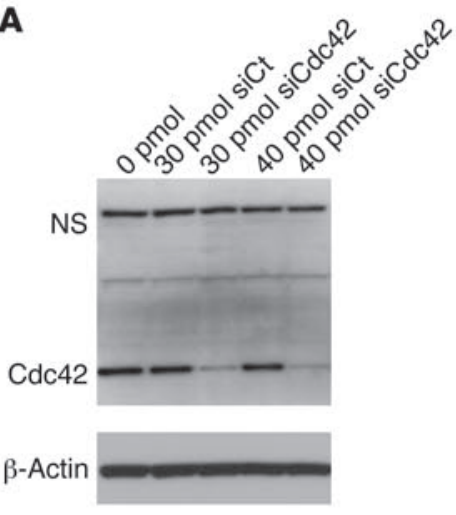

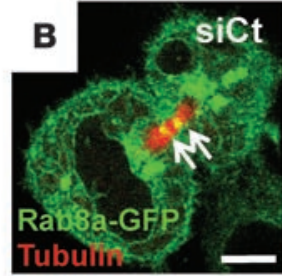
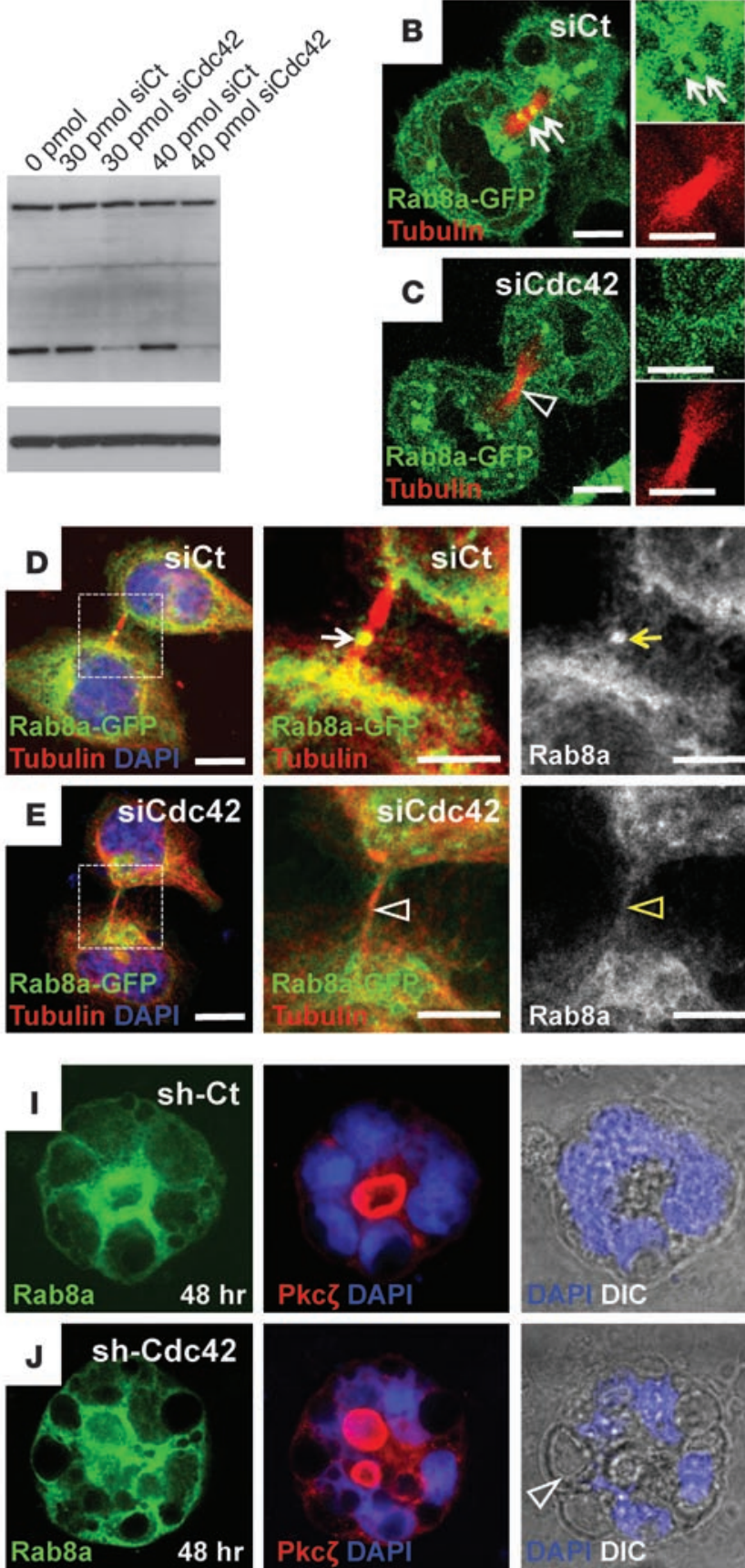
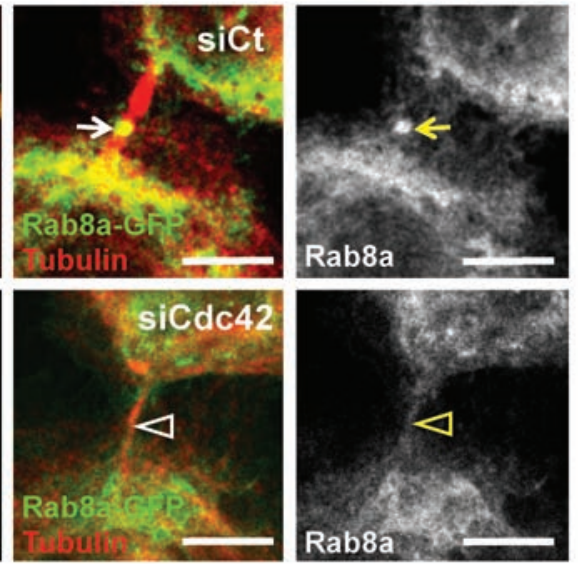
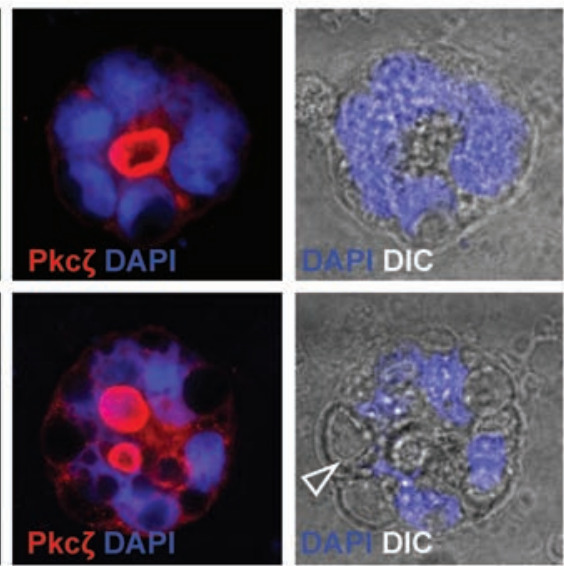

$\mathbf{F}$

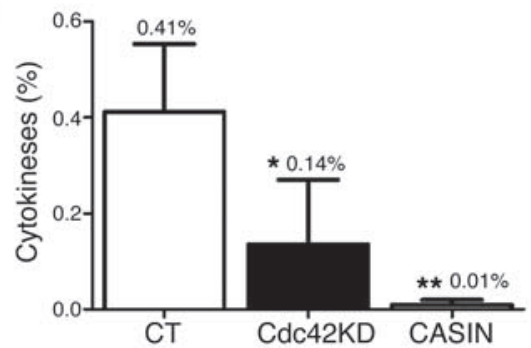

G

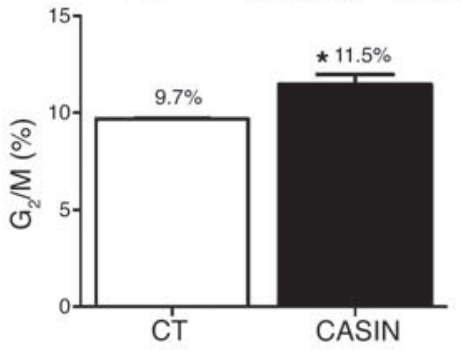

H

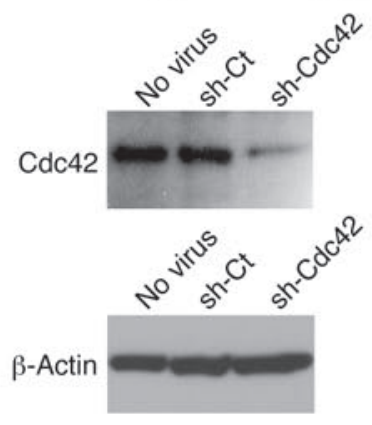

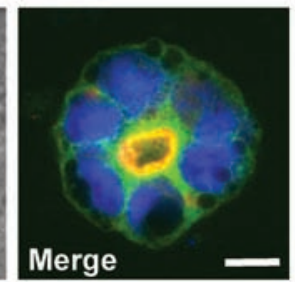

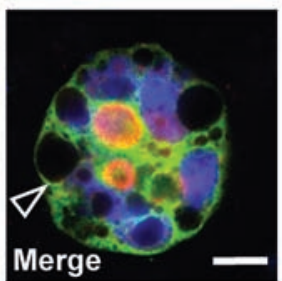

\section{Figure 7}

Cdc42 depletion affects midbody trafficking of Rab8a vesicle and cytokinesis. (A) Western blots confirmed Cdc42 knockdown by siRNA in Hela cells. (B and C) Live cell imaging showed that Rab8a-GFP (green) trafficked to the mcherry-tubulin-labeled midbody (red) during early cytokinesis in control cells but not in Cdc42-depleted cells. Arrows in B indicate enriched Rab8a at control midbody. Arrowhead in $\mathbf{C}$ indicates less Rab8a protein at midbody of Cdc42-depleted cells. (D and E) Rab8a-GFP localizes to the midbody (arrows in D) during late cytokinesis in control cells but not in Cdc42-depleted cells (arrowheads in E). (F) Quantification of cytokinesis in cells transfected with control siRNA, Cdc42 siRNA (Cdc42KD), and cells treated with $7 \mu \mathrm{M}$ CASIN. (G) FACS cell cycle analyses of CASIN-treated cells showed accumulation at the $\mathrm{G}_{2} / \mathrm{M}$ phase. (H) Western blots confirmed Cdc42 knockdown in Caco2 cells by a lentiviral shRNA particle. (I and J) Rab8a and Pkc $\zeta$ staining for control and Cdc42 knockdown Caco2 cysts. Arrowheads in $\mathbf{J}$ indicate large vacuoles where Rab8a was localized. Scale bars: $10 \mu \mathrm{m}$. ${ }^{\star} P<0.05$; ${ }^{\star \star} P<0.01$.

Cytokinesis and polarity establishment are closely linked processes. During epithelial morphogenesis in vitro in a $3 \mathrm{D}$ environment, distinct apical and basolateral membrane domains are frequently established at or immediately after the very first cell division $(11,22)$, which suggests that these processes are accomplished at the same time. Dynamic membrane traffic has long been recognized as a driving force for cytokinesis in lower eukaryotes (27-29), and the vibrant apical membrane recycling has also been well illustrated 


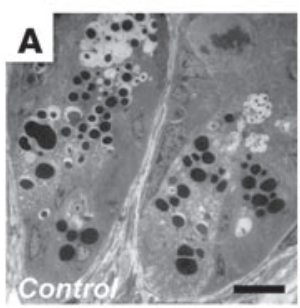

$\mathbf{E}$

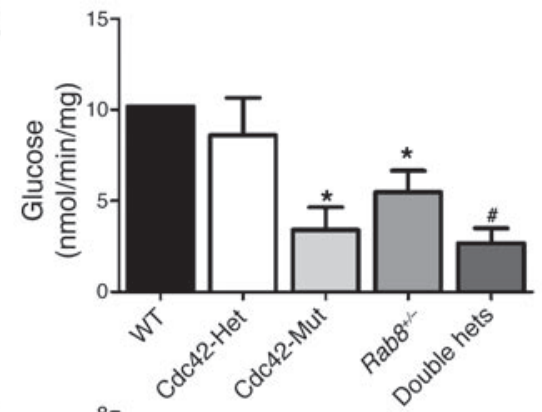

$\mathbf{F}$

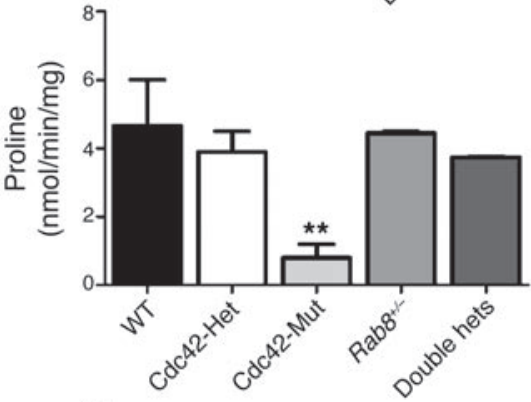

G

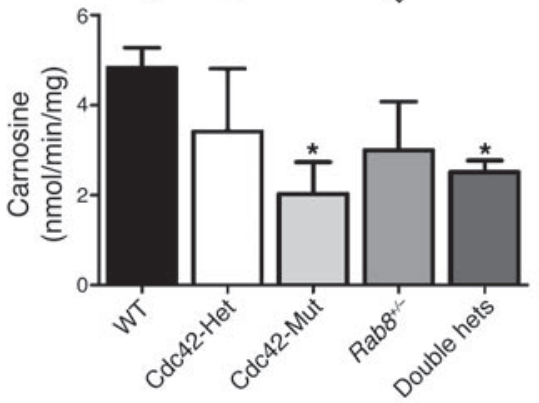

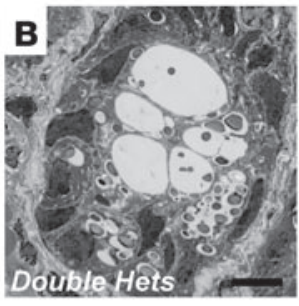

H
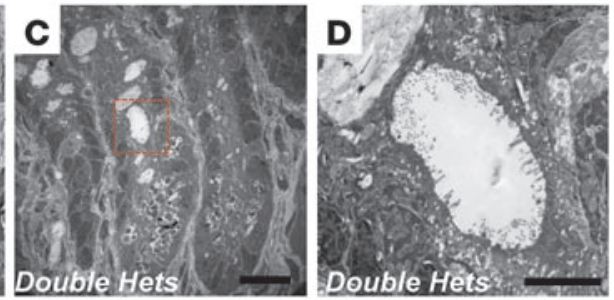

Cytokinesis of intestinal stem cell

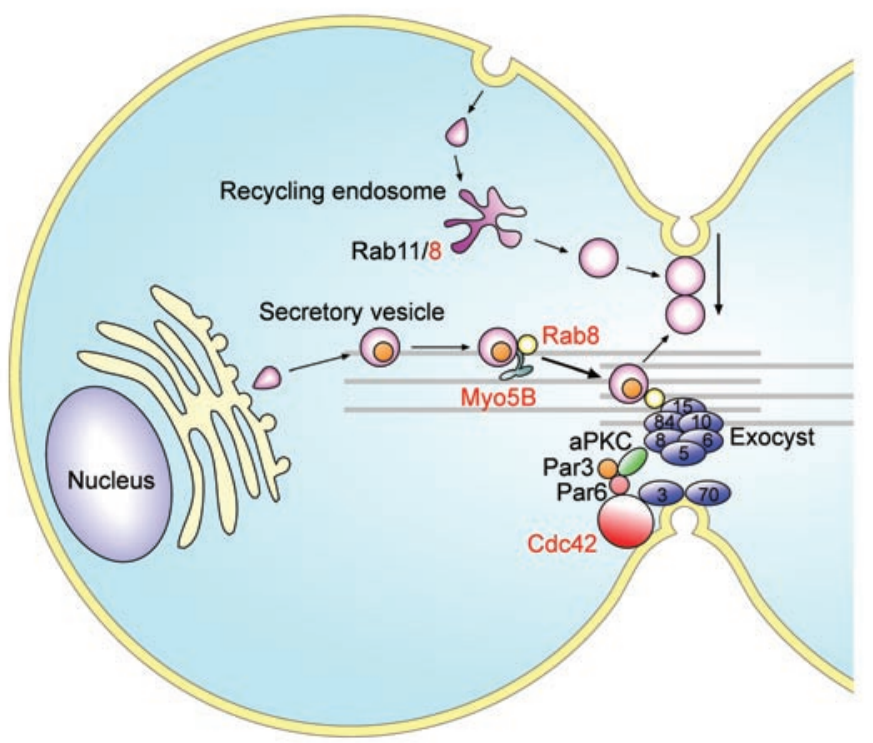

Figure 8

Cdc42 and Rab8a double heterozygous mice show abnormal crypt morphology and epithelial uptake. (A-D) TEM micrographs of control and double heterozygous crypts. Scale bars: $10 \mu \mathrm{m}(\mathbf{A}-\mathbf{C}) ; 500 \mathrm{~nm}$ (D). (E-G) Intestinal transporter uptake assays for glucose, proline, and carnosine. ${ }^{*} P<0.05$ compared with wild type; ${ }^{\#} P<0.05$ compared with wild type and single heterozygous; ${ }^{* *} P<0.01$ compared with wild type. (H) A model depicting the coordination of $\mathrm{Cdc} 42$ and Rab8a during intestinal stem cell division and epithelial morphogenesis. Cdc42, Rab8a, and Myo5B are shown in red to indicate their association with MVID. The exocyst, an octameric protein complex of Sec3, Sec5, Sec6, Sec8, Sec10, Sec15, Exo70, and Exo84, is indicated as blue circles at midbody.

in dividing mammalian cells (30). Rab small GTPases, in particular Rab11 and Rab8, localize mainly to the recycling endosome and exocytic vesicles and control protein trafficking to plasma membrane $(10,31)$. Indeed, disruption of Rab11a also disrupted the cleaving furrow formation in several model systems $(27,28)$, highlighting an essential role of these small GTPases in membrane addition and vesicle fusion at midbody. The fact that an epithelial defect was only observed in Rab8a knockout intestine but not kidney (4) further supported our view that the constant division of intestinal progenitor cells was more sensitive to the trafficking disturbance elicited by the loss of Rab8a. In our view, the basolat- eral trafficking (8-10) and de novo lumen formation defects (11) observed in Rab8a-deficient MDCK kidney cell line in vitro could be the direct consequences of abnormal cell division. Furthermore, most if not all polarity regulators within the Cdc42-Par-aPkc complex or its downstream effectors participate in spindle or midbody orientations $(22,32-39)$. Taken together, this is consistent with our findings that the cell division defect is the primary cause of epithelial abnormality upon loss of these small GTPases.

Collectively, we propose a model that Cdc42 establishes a protein complex containing Cdc42-Par3-Pkc' $\zeta$ and exocyst subunits (40) at midbody, which recruits the transport of Rab8 vesicles via Myo5b 
motor protein. The fusion of exocyst-tethered Rab8a vesicles at midbody facilitates cytokinesis and the establishment of prospective apical domain (model depicted in Figure 8H). On the other hand, the midbody and apical traffic of Rab8a deliver additional Par3 to reinforce the $\mathrm{Cdc} 42$ complex and amplify the apical determination signal (ref. 11 and Figure 8H). The increased Par3 level in Cdc42deficient intestines may reflect an attempt to activate $\mathrm{Cdc} 42$ in the mutant cells. Further genetic evidence is necessary to identify additional regulators of gut stem cell division and elucidate the molecular trafficking mechanisms essential for both stem cell renewal and epithelial morphogenesis.

\section{Methods}

Mice. $C d c 42^{\text {loxp }}$ (41) and Rab8 floxed (4) mice have been previously described. Lgr5CreER-IRES-EGFP (16), VillinCre (15), and Rosa-EYFP (42) mice were obtained from The Jackson Laboratory under the following strain names:

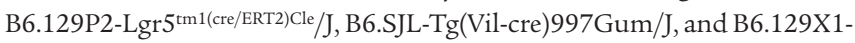

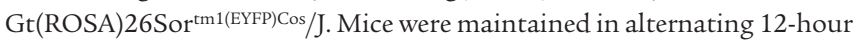
light/12-hour dark cycles on a regular diet at Rutgers University Animal Facility in Newark, New Jersey, USA.

For inducible gene ablation and lineage-tracing experiments, mice were intraperitoneally injected with $1 \mathrm{mg}$ tamoxifen (Sigma-Aldrich) dissolved in corn oil (Sigma-Aldrich). Mouse intestines were studied 1-3 weeks following the first injection. For each experiment, 3 to 6 littermate mice from each genotype were used.

Cell lines, plasmids, transfection, live cell imaging, and immunofluorescence staining. Human colonic epithelial $\mathrm{Caco} 2$ and human cervical adenocarcinoma Hela cell lines were purchased from ATCC and maintained according to ATCC instructions. Rab8a-EGFP and GST-JFC1 constructs were described previously (19).

Hela cells were cotransfected with Rab8a-EGFP, mcherry-tubulin plus SMARTpool siRNA against human Cdc42 (catalog no. L-005057-00-0005) or control siRNA (catalog no. D-001810-04-05) (Fisher Scientific) with DharmaFECT Duo Transfection Reagent. Live cell imaging was performed 24 hours after transfection. For staining, cells were fixed in $4 \%$ paraformaldehyde permeabilized with PBS with $0.5 \%$ Triton X-100, blocked with PBS plus $10 \%$ serum, and stained overnight with $\alpha$-tubulin antibody (1:200; Sigma-Aldrich) diluted in blocking buffer. Alexa Fluor 546-conjugated donkey anti-mouse secondary antibody (Invitrogen) was used to visualize tubulin staining along with green Rab8a-EGFP signals. Nuclei were stained with DAPI or TO-PRO-3 (Invitrogen). CASIN was a gift from Yi Zheng (Cincinnati Children's Hospital Medical Center, Cincinnati, Ohio, USA).

Lentivirus-mediated Cdc42 knockdown and Caco 2 cystogenesis. Procedures for in vitro $\mathrm{Caco} 2$ cystogenesis and cyst immunofluorescence staining have been described previously (6). To knockdown Cdc42 in Caco2 cells, human CDC42-specific lentiviral transduction particles (clone ID TRCN0000047628; Sigma-Aldrich) was used at 1-5 multiplicity of infection with $8 \mu \mathrm{g} / \mathrm{ml}$ Polybrene. Non-Target shRNA Control Transduction Particles (Sigma-Aldrich; catalog no. SHC203V) were used as negative controls.

Histology, confocal immunofluorescence, and TEM. H\&E staining, AP staining, Alcian blue staining, immunofluorescence, and EM analysis for gut tissues have been previously described (6,7). GFP staining for Lgr 5 CreER-EGFP intestinal tissues was performed on $9-\mu \mathrm{m}$ OCT-embedded frozen sections. Primary antibodies for immunofluorescence staining include E-cadherin (BD Transduction Laboratories; catalog no. 610182), DBAlectin (Vector Laboratories), lysozyme (Biogenex; catalog no. AR024-5R), pHH3 (Millipore; catalog no. 06-570), Villin (Santa Cruz Biotechnology Inc.; catalog no. sc-7672), EEA1 (Abcam; catalog no. 2900-100), $\alpha$-tubulin (Sigma Aldrich; catalog no. T9026), GFP (Invitrogen [catalog no. A11122] and Abcam [catalog no. ab6673]), Pkç (Santa Cruz Biotechnology Inc.; catalog no. sc-216G), Rab8a (BD Transduction Laboratories; catalog no. 610845), $\beta$-catenin (Cell Signaling; catalog no. 9587), laminin (Abcam; catalog no. 11575), Ki67 (Vector Laboratories; catalog no. VP-K451), BrdU (Accurate Chemical; catalog no. OBT0030G), $\mathrm{Na}^{+} / \mathrm{K}^{+}$-ATPase (a5; Developmental Studies Hybridoma Bank, University of Iowa, Iowa City, Iowa, USA). Fluorescence images were acquired using a Zeiss LSM 510 confocal microscope. Bright-field histology images were acquired using a Nikon TE2000 inverted microscope. TUNEL staining was performed as previously described (43).

Western blot, co-IP, and acetic acid-urea polyacrylamide gel electrophoresis. Procedures for Western blot have been described previously $(6,7)$. For co-IP, $1.5 \mathrm{mg}$ total protein lysates were incubated at $4^{\circ} \mathrm{C}$ for 3 hours with $20 \mu \mathrm{l}$ protein G Sepharose beads (GE Healthcare Life Sciences) conjugated with anti-Sec8 antibody. Beads were then washed 3 times in lysis buffer and once with PBS, heated at $70^{\circ} \mathrm{C}$ for 10 minutes in $4 \times$ LDS buffer (Invitrogen), and applied to $4 \%-12 \%$ SDS-PAGE NuPAGE gels (Invitrogen) for Rab8a Western blot analysis. Antibodies used for Western blots included Cdc42 (Cell Signaling; catalog no. 2462), G3PDH (Trevigen), Rab8a (BD Transduction Laboratory; catalog no. 610845), pPkç (Santa Cruz Biotechnology Inc.; catalog no. sc-271962), Par3 (Upstate-Millipore; catalog no. 07-330), $\beta$-actin (Cell Signaling; catalog no. 4967), Rabin8 (Abnova; catalog no. H00117177-B01), and Rab11a (US Biological; catalog no. R0009).

Defensin 5 expression levels were determined with acetic acid-urea polyacrylamide gel electrophoresis coupled with Western blotting, as previously described (44). Briefly, intestinal tissue lysates $(100 \mu \mathrm{g})$ were prepared in denaturing sample buffer (1M HCl, 9M urea, 5\% 2-mercaptoethanol) and separated by $12 \%$ AU-PAGE at $150 \mathrm{~V}$ for 2 hours. The separated proteins were transferred to nitrocellulose membrane in $0.7 \%$ acetic acid $/ 10 \%$ methanol at $180 \mathrm{~mA}$ for 10 minutes. Membranes were fixed in $0.05 \%$ glutaraldehyde in PBST for 1 hour, blocked in 5\% skimmed milk in PBST for 2 hours, and then probed with rabbit anti-defensin 5 (Abnova; 1:100 dilution) to confirm Defa5 expression levels.

Quantitative RT-PCR. Quantitative RT-PCR was performed as previously described $(6,7)$ with specific primers for mouse Lgr5, Bmi1, Msi1, Hopx, lysozyme, Mmp7, Pla2, and Defa5 (sequences are provided in Supplemental Table 1).

GST pull-down assay, Rab8a activation, and Cdc42 activation assay. GST-JFC1 fusion and GST proteins were expressed in BL21 E.coli (Invitrogen) and purified with glutathione-agarose beads (Sigma-Aldrich). For the GST pulldown assay, $2 \mathrm{mg}$ intestinal tissue lysates were incubated with GST proteinconjugated beads for 1 hour at $4{ }^{\circ} \mathrm{C}$. The beads were then washed with PBS 4 times and subjected to Western blot analysis for Rab8a. Cdc42 activation assays were performed using a kit from Millipore (catalog no. 17-441).

Cell cycle analysis by flow cytometry. After overnight treatment with $7 \mu \mathrm{M}$ CASIN, control and treated cells $\left(1-2 \times 10^{6}\right)$ were trypsinized and resuspended in PBS, fixed in cold ethanol at $4^{\circ}$ overnight, and stained with $40 \mu \mathrm{g} / \mathrm{ml}$ propidium iodide and $100 \mu \mathrm{g} / \mathrm{ml}$ RNase A in PBS for 30 minutes at $37^{\circ}$ before cell cycle analysis with a BD Biosciences FACSCalibur.

For imaging flow cytometry, cells were transfected with control siRNA, Cdc42 siRNA, or treated with $7 \mu \mathrm{M}$ CASIN before harvesting for propidium iodide staining as described above. An Amnis ImageStream (Amnis Corp.) imaging flow cytometer was used to capture high-resolution digital images in bright and fluorescent fields. Approximately $1 \times 10^{4}$ cells with a flow rate of 100 cells/second were analyzed, and cells with anaphase morphology were extracted using ImageStream IDEAS software.

Intestinal transporter assay. Intestinal uptake rates were determined as described previously $(45,46)$. Briefly, 1 -cm segments of the proximal small intestine were individually mounted and everted on grooved steel rods. Rods with the intestinal segment were preincubated at $37^{\circ} \mathrm{C}$ for 5 minutes in regular Krebs ringer buffer (KRB) and bubbled with 
$95 \% \mathrm{O}_{2} / 5 \% \mathrm{CO}_{2}$. For glucose uptake, the tissue was incubated at $37^{\circ} \mathrm{C}$ for 1 minute in a freshly made oxygenated solution of modified KRB (KRB with $103 \mathrm{mM} \mathrm{NaCl}$ ) containing $50 \mathrm{mM}$ D-glucose, $380 \mu \mathrm{M} \mathrm{D}-\left[{ }^{14} \mathrm{C}\right]$ glucose, and $0.2 \mu \mathrm{M} \mathrm{L}$ - $\left[{ }^{3} \mathrm{H}\right]$ glucose. $\mathrm{L}-\left[{ }^{3} \mathrm{H}\right]$ glucose was used to correct for adherent fluid and passive diffusion. Finally, all sleeves were rinsed for $20 \mathrm{sec}-$ onds in ice-cold KRB with stirring. For L-proline uptake, the segment was incubated in $50 \mathrm{mM} \mathrm{L}$-proline, $0.2 \mu \mathrm{M} \mathrm{L}-\left[{ }^{3} \mathrm{H}\right]$ proline, and $20 \mu \mathrm{M}$ inulin [carboxyl- ${ }^{14} \mathrm{C}$ ]; for L-carnosine uptake, the segment was incubated in $25 \mathrm{mM} \mathrm{L}$-carnosine, $0.9 \mu \mathrm{M} \mathrm{L}-\left[{ }^{3} \mathrm{H}\right]$ carnosine and $20 \mu \mathrm{M}$ inulin [carboxyl$\left.{ }^{14} \mathrm{C}\right]$ (to correct for adherent fluid).

Each 1-cm piece of intestine was transferred to a pre-weighed glass vial, weighed, and dissolved in $1 \mathrm{ml}$ of Solvable (PerkinElmer) at $37^{\circ} \mathrm{C}$ overnight. Following tissue digestion, a $10-\mathrm{ml}$ volume of Ecolume (MP Biomedicals) was added to each vial and vortexed, and ${ }^{3} \mathrm{H}$ and ${ }^{14} \mathrm{C}$ activity was determined with dual-label counting using a manually calibrated LS 6500 Multi-Purpose Scintillation Counter (Beckman Coulter). Quench curves were created by using the LS 6500 software and counting samples with a range of tissue weights, each spiked with the same amount of ${ }^{3} \mathrm{H}$, ${ }^{14} \mathrm{C}$, or ${ }^{3} \mathrm{H}$ plus ${ }^{14} \mathrm{C}$. Uptakes of nutrients are expressed as nanomoles per minute per milligram of wet weight of intestine.

Statistics. Morphometric analyses of intestinal villi and crypts were performed using Image J software. Data were analyzed using a 2-tailed Student's $t$ test. Mean values are shown in all graphs, with error bars representing SEM. A $P$ value less than 0.05 was considered significant.
Study approval. Mouse studies were performed with approval from the Rutgers University Institutional Animal Care and Use Committee.

\section{Acknowledgments}

We are grateful to Yi Zheng for providing the CASIN chemical inhibitor. This work was supported by the NIH/NIDDK (grant 5K01DK085194-03), the Charles and Johanna Busch Memorial Award (no. 659160), a Rutgers University Faculty Research Grant, Biological Sciences Departmental Funds (to N. Gao), National Institute of General Medical Sciences (grant 5RO1GM064690), the American Heart Association for Established Investigator Award (to W. Guo), the National Science Foundation (grant IOS-7223265), the Foundation of the University of Medicine \& Dentistry of New Jersey (grant PC44-11 to R.P. Ferraris), and an Institut Benjamin Delessert grant (to V. Douard). The authors thank Edward Bonder, Irina Bochkis, and Sebastian Rieck for helpful discussion of the manuscript.

Received for publication December 5, 2011, and accepted in revised form January 10, 2012.

Address correspondence to: Nan Gao, 195 University Ave., Boyden Hall 206, Newark, New Jersey 07102, USA. Phone: 973.353.5523; Fax: 973.353.5518; E-mail: ngao@andromeda.rutgers.edu.
1. Lake BD. Microvillus inclusion disease: specific diagnostic features shown by alkaline phosphatase histochemistry. J Clin Pathol. 1988;41(8):880-882.

2. Muller T, et al. MYO5B mutations cause microvillus inclusion disease and disrupt epithelial cell polarity. Nat Genet. 2008;40(10):1163-1165.

3. Roland JT, Bryant DM, Datta A, Itzen A, Mostov KE, Goldenring JR. Rab GTPase-Myo5B complexes control membrane recycling and epithelial polarization. Proc Natl Acad Sci US A. 2011;108(7):2789-2794.

4. Sato T, et al. The Rab8 GTPase regulates apical protein localization in intestinal cells. Nature. 2007; 448(7151):366-369.

5. Chavrier P, Vingron M, Sander C, Simons K, Zerial M. Molecular cloning of YPT1/SEC4-related cDNAs from an epithelial cell line. Mol Cell Biol. 1990; 10(12):6578-6585

6. Gao N, Kaestner KH. Cdx2 regulates endo-lysosomal function and epithelial cell polarity. Genes Dev. 2010;24(12):1295-1305.

7. Gao N, White P, Kaestner KH. Establishment of intestinal identity and epithelial-mesenchymal signaling by Cdx2. Dev Cell. 2009;16(4):588-599.

8. Huber LA, Pimplikar S, Parton RG, Virta H, Zerial $\mathrm{M}$, Simons K. Rab8, a small GTPase involved in vesicular traffic between the TGN and the basolateral plasma membrane. J Cell Biol. 1993;123(1):35-45.

9. Ang AL, Folsch H, Koivisto UM, Pypaert M, Mellman I. The Rab8 GTPase selectively regulates AP$1 \mathrm{~B}$-dependent basolateral transport in polarized Madin-Darby canine kidney cells. J Cell Biol. 2003; 163(2):339-350.

10. Ang AL, et al. Recycling endosomes can serve as intermediates during transport from the Golgi to the plasma membrane of MDCK cells. J Cell Biol. 2004; 167(3):531-543.

11. Bryant DM, Datta A, Rodriguez-Fraticelli AE, Peranen J, Martin-Belmonte F, Mostov KE. A molecular network for de novo generation of the apical surface and lumen. Nat Cell Biol. 2010;12(11):1035-1045.

12. Sato T, et al. Paneth cells constitute the niche for Lgr5 stem cells in intestinal crypts. Nature. 2010; 469(7330):415-418.

13. Snippert HJ, et al. Intestinal crypt homeostasis results from neutral competition between symmetrically dividing Lgr5 stem cells. Cell. 2010;
143(1):134-144.

14. Lin D, Edwards AS, Fawcett JP, Mbamalu G, Scott JD, Pawson T. A mammalian PAR-3-PAR-6 complex implicated in Cdc42/Rac1 and aPKC signalling and cell polarity. Nat Cell Biol. 2000;2(8):540-547.

15. Madison BB, Dunbar L, Qiao XT, Braunstein K, Braunstein E, Gumucio DL. Cis elements of the villin gene control expression in restricted domains of the vertical (crypt) and horizontal (duodenum, cecum) axes of the intestine. J Biol Chem. 2002; 277(36):33275-33283.

16. Barker $\mathrm{N}$, et al. Identification of stem cells in small intestine and colon by marker gene Lgr5. Nature. 2007; 449(7165):1003-1007.

17. Tian $\mathrm{H}$, et al. A reserve stem cell population in small intestine renders Lgr5-positive cells dispensable. Nature. 2011;478(7368):255-259.

18. Takeda N, Jain R, LeBoeuf MR, Wang Q, Lu MM, Epstein JA. Interconversion between intestinal stem cell populations in distinct niches. Science. 2011; 334(6061):1420-1424.

19. Knodler A, et al. Coordination of Rab8 and Rab11 in primary ciliogenesis. Proc Natl Acad Sci U S A. 2010; 107(14):6346-6351.

20. Hattula K, Furuhjelm J, Tikkanen J, Tanhuanpaa K, Laakkonen P, Peranen J. Characterization of the Rab8-specific membrane traffic route linked to protrusion formation. J Cell Sci. 2006; 119(pt 23):4866-4877.

21. Guo W, Roth D, Walch-Solimena C, Novick P. The exocyst is an effector for Sec4p, targeting secretory vesicles to sites of exocytosis. EMBO J. 1999 ; 18(4):1071-1080.

22. Jaffe AB, Kaji N, Durgan J, Hall A. Cdc42 controls spindle orientation to position the apical surface during epithelial morphogenesis. J Cell Biol. 2008; 183(4):625-633.

23. Akbar H, et al. Genetic and Pharmacologic Evidence Shows That Cdc42 GTPase Plays a Central Role in the Regulation of Both GPVI- and NonGPVI-Dependent Activation of Platelets. 51th ASH Annual Meeting and Exposition. New Orleans, Louisiana, USA. December 5-8, 2009.

24. Rincon S, Coll PM, Perez P. Spatial regulation of Cdc42 during cytokinesis. Cell Cycle. 2007; 6(14):1687-1691.
25. Drechsel DN, Hyman AA, Hall A, Glotzer M. A requirement for Rho and $\mathrm{Cdc} 42$ during cytokinesis in Xenopus embryos. Curr Biol. 1997;7(1):12-23.

26. Bielak-Zmijewska A, Kolano A, Szczepanska K, Maleszewski M, Borsuk E. Cdc42 protein acts upstream of IQGAP1 and regulates cytokinesis in mouse oocytes and embryos. Dev Biol. 2008; 322(1):21-32

27. Skop AR, Bergmann D, Mohler WA, White JG. Completion of cytokinesis in C. elegans requires a brefeldin A-sensitive membrane accumulation at the cleavage furrow apex. Curr Biol. 2001; 11(10):735-746.

28. Riggs B, et al. Actin cytoskeleton remodeling during early Drosophila furrow formation requires recycling endosomal components Nuclear-fallout and Rab11. J Cell Biol. 2003;163(1):143-154.

29. Albertson R, Riggs B, Sullivan W. Membrane traffic: a driving force in cytokinesis. Trends Cell Biol. 2005; 15(2):92-101.

30. Hobdy-Henderson KC, Hales CM, Lapierre LA Cheney RE, Goldenring JR. Dynamics of the apical plasma membrane recycling system during cell division. Traffic. 2003;4(10):681-693.

31. Ullrich O, Reinsch S, Urbe S, Zerial M, Parton RG. Rab11 regulates recycling through the pericentriolar recycling endosome. J Cell Biol. 1996; 135(4):913-924.

32. Gotta M, Abraham MC, Ahringer J. CDC-42 controls early cell polarity and spindle orientation in C. elegans. Curr Biol. 2001;11(7):482-488.

33. Ma C, et al. Cdc42 activation couples spindle positioning to first polar body formation in oocyte maturation. Curr Biol. 2006;16(2):214-220.

34. Kieserman EK, Wallingford JB. In vivo imaging reveals a role for $\mathrm{Cdc} 42$ in spindle positioning and planar orientation of cell divisions during vertebrate neural tube closure. J Cell Sci. 2009; 122(pt 14):2481-2490.

35. Mitsushima M, Toyoshima F, Nishida E. Dual role of Cdc42 in spindle orientation control of adherent cells. Mol Cell Biol. 2009;29(10):2816-2827.

36. Durgan J, Kaji N, Jin D, Hall A. Par6B and atypical PKC regulate mitotic spindle orientation during epithelial morphogenesis. J Biol Chem. 2011; 286(14):12461-12474. 
37. Qin Y, Meisen WH, Hao Y, Macara IG. Tuba, a Cdc42 GEF, is required for polarized spindle orientation during epithelial cyst formation. J Cell Biol. 2010; 189(4):661-669.

38. Hao Y, et al. Par3 controls epithelial spindle orientation by aPKC-mediated phosphorylation of apical Pins. Curr Biol. 2010;20(20):1809-1818.

39. Rodriguez-Fraticelli AE, et al. The Cdc42 GEF Intersectin 2 controls mitotic spindle orientation to form the lumen during epithelial morphogenesis. J Cell Biol. 2010;189(4):725-738.

40. Das A, Guo W. Rabs and the exocyst in ciliogenesis, tubulogenesis and beyond. Trends Cell Biol. 2011;
21(7):383-386.

41. Wu X, et al. Cdc42 controls progenitor cell differentiation and beta-catenin turnover in skin. Genes Dev. 2006;20(5):571-585.

42. Srinivas $S$, et al. Cre reporter strains produced by targeted insertion of EYFP and ECFP into the ROSA26 locus. BMC Dev Biol. 2001;1:4.

43. Gao N, et al. The nuclear pore complex protein Elys is required for genome stability in mouse intestinal epithelial progenitor cells. Gastroenterology. 2011; 140(5):1547-1555.

44. Cunliffe RN, Rose FR, Keyte J, Abberley L, Chan WC, Mahida YR. Human defensin 5 is stored in pre- cursor form in normal Paneth cells and is expressed by some villous epithelial cells and by metaplastic Paneth cells in the colon in inflammatory bowel disease. Gut. 2001;48(2):176-185.

45. Roche M, et al. Marked changes in endogenous antioxidant expression precede vitamin A-, C-, and E-protectable, radiation-induced reductions in small intestinal nutrient transport. Free Radic Biol Med. 2011;50(1):55-65.

46. Douard V, et al. Dietary fructose inhibits intestinal calcium absorption and induces vitamin D insufficiency in CKD. J Am Soc Nephrol. 2010; 21(2):261-271. 